\title{
Unfolded protein response and scaffold independent pheromone MAP kinase signalling control Verticillium dahliae growth, development and plant pathogenesis
}

Jessica Starke1, Rebekka Harting ${ }^{1}$, Isabel Maurus ${ }^{1}$, Rica Bremenkamp${ }^{1}$, James W. Kronstad$^{2}$, Gerhard H. Braus ${ }^{1 *}$

${ }^{1}$ Department of Molecular Microbiology and Genetics, Institute of Microbiology and Genetics and Göttingen Center for Molecular Biosciences (GZMB), University of Göttingen, Grisebachstr. 8, D-37077 Göttingen, Germany

2Department of Microbiology and Immunology, Michael Smith Laboratories, University of British Columbia, Vancouver, BC, V6T 1Z4, Canada

*Corresponding author: Gerhard H. Braus

Email: gbraus@gwdg.de 


\section{Summary}

- Development and virulence of the vascular plant pathogen Verticillium dahliae are connected and depend on a complex interplay between the unfolded protein response, a Ham5 independent pheromone MAP kinase module and formation of precursors for oxylipin signal molecules.

- Genes coding for the unfolded protein response regulator Hac1, the Ham5 MAPK scaffold protein, and the oleate $\Delta 12$-fatty acid desaturase Ode1 were deleted and their functions in growth, differentiation, and virulence on plants were studied using genetic, cell biology, and plant infection experiments.

- The unfolded protein response transcription factor Hac1 is required for initial root colonization, fungal conidiation and propagation inside the host and is essential for resting structure formation. Microsclerotia development, growth and virulence require the pheromone response MAPK pathway, but without the Ham5 scaffold function. Single ER-associated enzymes for linoleic acid production make important contributions to fungal growth but have only a minor impact on the pathogenicity of $V$. dahliae.

- Fungal growth, sporulation, dormant structure formation and plant infection require a network of the Hac1-regulated unfolded protein response, a scaffoldindependent pheromone response MAPK pathway and formation of precursors for signalling. This network includes interesting targets for disease management of the vascular pathogen $V$. dahliae.

Keywords: unfolded protein response, MAPK signalling, MAPK scaffold, oleate $\triangle 12-$ fatty acid desaturase, Verticillium dahliae, plant pathogen

\section{Introduction}

Verticillium dahliae is a soil-borne asexual ascomycete causing vascular wilting disease in a broad range of plants including high value crops (Pegg \& Brady, 2002; Luo et al., 2014). Unfavourable conditions induce the formation of highly resistant, black melanized microsclerotia as characteristic dormant structures which persist in the soil and can overwinter for at least 14 years (Wilhelm, 1955; Griffiths, 1970; Pegg \& Brady, 2002). The fungus germinates upon recognition of an appropriate host and enters the plant preferably via natural root wounds, root tips or lateral root hairs (Fitzell et al., 1980; Eynck et al., 2007; Vallad \& Subbarao, 2008; Su et al., 2018). Hyphae 
grow from cortical cells towards the central cylinder and some of them successfully reach the xylem (Klosterman et al., 2009). Asexual spores are formed and spread within the vascular system via the transpiration stream (Klosterman et al., 2009). Colonization of tissues neighbouring the xylem correlates with induction of disease symptoms (Fradin \& Thomma, 2006). Limited nutrient availability in the dying host or in plant debris induces the formation of microsclerotia for persistence (Fradin \& Thomma, 2006). During the infection cycle signals from the host environment induce differentiated fungal development and activate specific responses to enable colonization and suppression of the plants immune system.

The unfolded protein response (UPR) pathway monitors protein folding and secretion capacities in the endoplasmic reticulum (ER) lumen and mediates expression of genes involved in ER stress relief (Kozutsumi et al., 1988; Kohno et al., 1993; Hetz, 2012; Heimel, 2015). Sensing of un- or misfolded proteins in the ER lumen depends on transmembrane sensors as Ire1 described in yeast (Mori et al., 1993; Okamura et al., 2000). The cytoplasmic endoribonuclease domain of Ire1 is responsible for unconventional splicing of the mRNA encoding the bZIP transcription factor Hac1 (Sidrauski \& Walter, 1997; Gonzalez et al., 1999). Splicing of the uninduced HAC1 (HAC14) mRNA results in the induced HAC1 (HAC1') mRNA variant. The HAC1' mRNA is translated into the Hac1 protein which regulates UPR target genes, encoding, for example, chaperones, glycosylation enzymes, proteins required for vesicle transport, lipid biosynthesis or regulators for adaptation of ER size (Cox et al., 1993; Mori et al., 1996, 1998; Kaufman, 1999; Travers et al., 2000; Conn, 2011; Hetz, 2012).

The role of the UPR transcription factor Hac1 homologs and orthologues varies within human or plant pathogenic fungal species (Krishnan \& Askew, 2014). The ER stress response mechanism of the opportunistic human pathogenic yeast Candida glabrata is regulated in an Ire1-dependent decay independently of Hac1 (Kimmig et al., 2012; Miyazaki et al., 2013). This results in splicing of various ER-associated mRNAs by Ire1 to cope with ER stress (Heimel et al., 2013; Miyazaki et al., 2013). Necrotrophic plant ascomycetes such as the rice blast fungus Magnaporthe oryzae require $\mathrm{Hac} 1$ and the UPR for conidia formation as well as for penetration and invasive hyphae growth during plant infection (Tang et al., 2015), whereas in Alternaria brassicicola the UPR is required for resistance against plant antimicrobial compounds and virulence, but is not involved in plant penetration and initial colonization (Joubert et al., 2011; Guillemette et al., 2014). The UPR pathway regulates virulence specific genes in the dimorphic 
basidiomycete corn smut fungus Ustilago maydis (Heimel et al., 2010; Hampel et al., 2016; Pinter et al., 2019). In this fungus a cross-regulation of the UPR pathway and the pheromone response mitogen-activated protein kinase (MAPK) pathway was described. Pathogenicity of the smut fungus depends on activation of the UPR after invasion of the plant surface (Heimel et al., 2010, 2013). When activated prior to entering the plant cell, the UPR inhibits filamentous growth and virulence by reduction of the pheromone response MAPK pathway activity through Rok1-dependent dephosphorylation of the MAPK Kpp2 (Schmitz et al., 2019).

The pheromone response MAPK pathway was originally described in Saccharomyces cerevisiae for a-cell- $\alpha$-cell fusion during mating where the core module consists of the Ste50 adaptor, the Ste11 MAP kinase kinase kinase (MAP3K), the Ste7 MAP kinase kinase (MAP2K), and the Fus3 MAP kinase (MAPK). A phosphorylation signal is sequentially transferred with the help of the scaffold protein Ste5 to regulate downstream targets such as the transcription factor Ste12 (Alvaro \& Thorner, 2016). Components of this pheromone MAPK core can also participate in other Ste5 independent MAPK pathways, such as the starvation mediated filamentous growth pathway of yeast comprised of the Ste50 adaptor, the MAP3K Ste11 and MAP2K Ste7, and the MAPK Kss1 (Cullen et al., 2012). The pheromone response MAPK pathway plays an important role in the pathogenicity of numerous filamentous fungi (Jiang et al., 2018).

The $V$. dahliae pheromone response MAPK pathway is essential for pathogenicity and includes the upstream adaptor Vste50, Ste11 (MAP3K), Mek2 (Vst7; MAP2K) corresponding to yeast Ste7, Vmk1 (MAPK) corresponding to Fus3 and the downstream Ste12-like transcription factor Vph1 (Rauyaree et al., 2005; Qi et al., 2016; Sarmiento-Villamil et al., 2018; Li et al., 2019; Yu et al., 2019). Vmk1, Mek2, Ste11, and Vste50-deficient mutant display defects in microsclerotia development, whereas VPH1 deletion strains are unaffected. Oxygenated polyunsaturated fatty acids (oxylipins) are fungal hormones, which modulate fungal development, pathogenicity and mycotoxin production (Fischer \& Keller, 2016). Oxylipins can trigger the biosynthesis of lipid metabolites with functions in plant host colonization, and can manipulate the host lipid metabolism and alter plant defence responses presumably by mimicking endogenous signal molecules. The interplay between plant and fungal oxylipins can direct the outcome of the fungus-plant interaction (Brodhun \& Feussner, 2011). The major precursor of fungal oxylipins is the polyunsaturated fatty acid linoleic 
acid $(18: 2 \Delta 9,12)$. Linoleic acid is synthesized by oleate $\Delta 12$-fatty acid desaturases such as OdeA from oleic acid (18:1 $\Delta 9)$ by introduction of a second double bond into the carbon chain at position 12 from the carboxy-terminus (Uttaro, 2006). OdeA is required for asexual and sexual development as well as the formation of resting structures in different Aspergilli (Calvo et al., 2001; Chang et al., 2004; Wilson et al., 2004).

In this study, we analysed the impact of the unfolded protein response and the pheromone response MAPK pathway on the development and pathogenicity of the vascular plant pathogen $V$. dahliae. We show that a functional UPR is required for successful colonization of its host. The UPR regulator Hac1 is essential for the formation of dormant structures, which is critical for the ability of the fungus to persist in the soil for many years and to successfully re-establish the disease in the next season. A functional UPR also contributes to the growth and conidiation of $V$. dahliae. The UPR might crosstalk with the pheromone response MAPK pathway, which does not require the Ham5 scaffold protein (Dettmann et al., 2014; Jonkers et al., 2014; Frawley et al., 2018) to promote $V$. dahliae resting structure formation, growth and virulence. The ER-associated oleate $\Delta 12$-fatty acid desaturase producing linoleic acid as precursor of oxylipin hormones primarily promotes fungal growth with only minor impacts on pathogenicity of $V$. dahliae. This analysis suggests an intricate interplay between UPR and pheromone MAPK signalling for fungal growth, development and virulence in the vascular pathogen $V$. dahliae.

\section{Material and Methods}

V. dahliae JR2 (Fradin et al., 2009) was used as wildtype for construction of all Verticillium strains used in this study. Southern hybridizations of constructed strains are shown in Supporting Information Figures S1, S2 and S3. All Verticillium strains are listed in Supporting Information Table S1, primers in Table S2 and plasmids in Table S3. Methods for bacterial and fungal cultivation and strain construction are described in Supporting Information Methods S1.

\section{Quantification of growth and developmental structures}

Verticillium growth was quantified as colony diameter over time. Melanization of colony centres was quantified by determination of the brightness factor using ImageJ software. Conidia were quantified from liquid SXM cultures. For details see Supporting Information Methods S1. 


\section{Confocal microscopy}

Localization of Ode1-GFP was examined by confocal fluorescence microscopy by inoculation of 50 000-100 000 freshly harvested spores in $300 \mu \mathrm{l}$ liquid PDM per well in $\mu$-slide 8 well microscopy chambers (ibidi) and incubation at $25^{\circ} \mathrm{C}$ for the indicated time. Hyphal morphology and subcellular localization were observed with a PlanNeofluar 100x/1.4 oil objective (Zeiss, 300 ms exposure time for GFP signals, 800 ms exposure time for RFP signals). Vacuoles were visualized by fluorescence microscopy after staining with 0.3 $\mu$ I FM4-64 Dye (Thermo Fisher Scientific) for one hour.

\section{Protein and nucleic acid purification and hybridization}

RNA was purified using the Direct-zol RNA MiniPrep Kit. Reverse transcription of RNA was performed using the QuantiTect Reverse Transcription Kit (Qiagen). Genomic DNA isolation, Southern hybridization, protein extraction and immunoblots were performed according to standard protocols. For details see Supporting Information Methods S1.

\section{Quantification of gene expression}

Expression levels of $H A C 1$ were quantified relative to the reference genes histone $H 2 A$ (VDAG_JR2_Chr4g01430a) and EIF2B (VDAG_JR2_Chr4g00410a) by qRT PCR. For details see Supporting Information Methods S1.

\section{Arabidopsis thaliana root colonization assay}

The root colonization assay was modified from Tran et al., 2014. Three-week-old A. thaliana (Col-0) plants were infected with conidial suspensions ( $\left.1 \times 10^{5} \mathrm{spores} / \mathrm{ml}\right)$ and fluorescence microscopy of the roots was conducted at the indicated time points. For details see Supporting Information Methods S1.

\section{Plant infection experiments}

Ten-day-old seedlings of Solanum lycopersicum (Moneymaker) were infected by root dipping into a solution of $1 \times 10^{7}$ conidiospores $/ \mathrm{ml}$ and disease symptoms were scored after $21 \mathrm{~d}$. Discoloration of the hypocotyl was determined by observation of cross sections. To test for fungal outgrowth from infected plants, stems were harvested, surface sterilized and incubated on PDM plates supplemented with chloramphenicol $(100 \mu \mathrm{g} / \mathrm{ml})$ at $25^{\circ} \mathrm{C}$ for $7 \mathrm{~d}$. For details see Supporting Information Methods S1.

\section{Sequence analyses}

The online databases National Center for Biotechnology Information (NCBI; Geer et al., 2010) and Ensembl Fungi (Kersey et al., 2018) were used for BLAST searches. 
Verticillium gene predictions, accession numbers, and sequences were obtained from Ensembl Fungi. The web server RNAfold (Zuker, 2003) was used for determination of secondary structures in mRNAs. Prediction of protein domains was performed using the InterPro website (http://www.ebi.ac.uk/Tools/pfa/iprscan; Jones et al., 2014). Presence of nuclear localization signals was analysed using the online databases cNLS mapper (http://nls-mapper.iab.keio.ac.jp/cgi-bin/NLS_Mapper_form.cgi Kosugi et al., 2009), and DeepLoc-1.0 (Almagro Armenteros et al., 2017; http://www.cbs.dtu.dk/services/DeepLoc/index.php). Multiple alignment of protein sequences was performed with ClustalW (Thompson et al., 1994) or Muscle (Edgar, 2004) algorithms in MEGA6.0 software (Tamura et al., 2013). Phylogenetic analysis was conducted using Maximum likelihood tree calculations with MEGA6.0 software.

\section{Results}

\section{The $V$. dahliae unfolded protein response pathway regulator Hac1 supports} fungal growth and is essential for resting structure development

The function of the UPR pathway regulator Hac1 for growth, development or virulence has not yet been analysed in fungal plant vascular pathogens. The UPR is important because it monitors secretion capacity and ER protein folding. In particular, the controlled formation of secreted effector proteins is critical for fungal differentiation processes and colonization-related adaptation to provide appropriate responses to different environmental signals, which are perceived at the cell membrane, transduced to the nucleus and then change ER mediated secretion.

A HAC1 homolog encoding the UPR transcription factor was identified in $V$. dahliae by BLAST search. The HAC1 gene carries a 53 nucleotides (nt) conventional intron and an additional non-conventional $20 \mathrm{nt}$ intron. Splicing of the conventional intron results in a HAC1u transcript of 1581 nt encoding a protein with 526 aa. Additional splicing of the non-conventional intron results in $H A C 1^{i}$ with an altered reading frame of $1254 \mathrm{nt}$ encoding for the 417 aa Hac1 protein. Expression of the larger HAC1u as well as the smaller $H A C 1^{i}$ splice variants were verified by characterizing amplified cDNA sequences. Only $H A C 1^{i}$ transcripts encoding Hac1 were found in the presence of ER stress mediated by DTT (induced conditions), whereas without DTT (uninduced conditions) both $\mathrm{HAC1}^{u}$ and smaller $\mathrm{HAC1}^{i}$ were identified (Fig. 1a-c, Supporting Information Table S4). 
The length of $20 \mathrm{nt}$ for the unconventionally spliced intron of $V$. dahliae HAC1 is similar to other filamentous ascomycetes (20-26 nt), but smaller than in the dimorphic basidiomycete $U$. maydis (65 nt) or in budding yeast (252 nt) (Fig. 1b). Unconventional splicing of mRNAs of $H A C 1$ homologs requires the cytosolic endoribonuclease domain of the ER membrane resident sensor Ire1. This domain recognizes the conserved consensus splice sites 5'-CNG'CNGN-3' (Hooks \& Griffiths-Jones, 2011a). 5' and 3' intron-exon-borders of the 20 nt intron from V. dahliae HAC1 are conserved (Fig. 1b). Similar to Hac1 splice sites of different organisms, the consensus splice site of the unconventional $V$. dahliae HAC1 intron was predicted to form a characteristic twin stem-loop secondary structure (Fig. 1c).

The two HAC1 splice variants encode proteins with identical $\mathrm{N}$ - but different $\mathrm{C}$-terminal regions. The shared N-terminal 268 aa region includes the NLS motif and the bZIP domain (Fig. 1a). The remaining C-termini of the deduced larger 526 aa Hac1 ${ }^{\mathrm{u}}$ protein of $58 \mathrm{kDa}$ and the smaller 417 aa Hac1 protein of $44 \mathrm{kDa}$ are unique (Fig. 1a, the amino acid sequence of Hac1 is shown in Table S5). A phylogenetic analysis revealed similarities between the $V$. dahliae Hac1 protein encoded by the unconventionally spliced mRNA HAC1 ${ }^{i}$ to described UPR regulatory proteins in other fungi and to human XBP1 (X-box binding protein) (Fig. 1d). Proteins from V. dahliae and T. reesei cluster in one subclade. The Hac1 protein of $S$. cerevisiae showed higher similarity to the $U$. maydis Cib1 protein than to $V$. dahliae Hac1.

A deletion strain was constructed to analyse the role of HAC1 in the development and virulence of $V$. dahliae. This mutant strain was used for ectopic reintegration of either the entire HAC1 gene (HAC1-C) or one of the two mRNA HAC1 variants (Fig. 1a) fused to $H A$ at the 3 '-end and under control of the native promoter and terminator. Expression levels of induced and uninduced HAC1 mRNA variants were examined with the same primer pair in the constructed strains in comparison to wildtype and $\triangle H A C 1$. The HAC1-C complementation strain displayed wildtype-like HAC1 expression levels, whereas expression was reduced to less than $40 \%$ in $H A C 1{ }^{u_{-}} H A$, and even to $\sim 60 \%$ in $H A C 1^{i}-H A$ (Fig. 2a). Immunoblot analysis was performed to investigate whether both HAC1 mRNA variants were translated into Hac1 ${ }^{\mathrm{u}}-\mathrm{HA}$ and $\mathrm{Hac1}$-HA fusion proteins. A band at $\sim 70 \mathrm{kDa}$ instead of the predicted $46 \mathrm{kDa}$ was obtained for $H A C 1^{-}-H A$, expressing the unconventionally spliced $H A C 1$ mRNA variant, whereas there were no signals for the $H A C 1^{u}-H A$ strain containing the mRNA variant where only the conventional intron was spliced (Fig. 2b). 
Alterations in growth and development of the constructed strains ex planta were compared to wildtype. $\triangle H A C 1$ colonies produce less aerial mycelium and appear more transparent. Under non-stress conditions reduced growth was observed for $\triangle H A C 1$ (15\%) and HAC1'-HA (10\%) in comparison to the wildtype strain (Fig. 2c, d). Under DTT-induced ER stress conditions growth of $\triangle H A C 1$ was decreased (18\% lower), whereas $H A C 1{ }^{i}-H A$ displayed relatively increased colony diameters (17\% higher) compared to wildtype. The expression of the ectopically integrated HAC1 gene in $H A C 1-C$ or the $H A C 1$ gene lacking the conventional intron in $H A C 1_{-}-H A$ in the deletion background resulted in wildtype-like growth under stress- and non-stress conditions. This suggests, that presence of the unconventionally spliced HAC1 mRNA in the HAC1'-HA strain enables a more efficient response to ER stress.

For $\triangle H A C 1$ no melanization of the colony centres was observed during growth on any tested medium. Cross sections and microscopy of fungal material from $\triangle H A C 1$ colonies grown on different media revealed the absence of microsclerotia, whereas ectopic integration resulted in increased resting structure occurrence for the $H A C 1-C$ and $H A C 1^{i}-H A$ strains as exemplified for minimal medium with sucrose as carbon source (Fig. 2c). Wildtype-like microsclerotia frequencies were observed in the $H A C 1 u_{-} H A$ strain. The absence of microsclerotia in $\triangle H A C 1$ and vice versa increased melanization in $H A C 1^{i}-H A$ corroborate a regulatory function of $H A C 1$ in resting structure formation.

\section{Virulence of $V$. dahliae depends on the unfolded protein response transcription} factor Hac1

As mentioned above, the unfolded protein response controls the capacity and quality of ER-mediated secretion. It is currently unknown whether the UPR regulator Hac1 is important for fungal pathogens that colonize plant roots. Therefore, we compared root colonization by a UPR defective $V$. dahliae HAC1 deletion strain versus the wildtype strain. A HAC1 deletion strain expressing ectopically integrated GFP under control of the gpdA promoter was constructed for monitoring colonization. Three-week-old Arabidopsis thaliana plants inoculated with the same number of spores obtained from $\triangle H A C 1 O E-G F P$ and wildtype carrying the same construct (WT OE-GFPNAT) were compared. Fungal colonization behaviour was studied after five to seven days. Overall, less hyphae on the root surface were present for the $\triangle H A C 1$ OE-GFP strain compared to wildtype (Fig. 3a). The formation of swollen hyphae and a change in growth direction 
indicate penetration sites. These features were observed in the absence of $H A C 1$ similarly to wildtype, as was hyphal growth after invasion of the root outer layer. Therefore, $V$. dahliae HAC1 is not required for penetration of the root and the mutant is not blocked directly after invasion of the root cortex, but HAC1 supports the first contact with the host, which is the initial colonization of the root surface.

$V$. dahliae forms conidiospores within the plant vascular system for spreading and systemic colonization. The HAC1 deletion strain displayed significantly reduced conidiospore numbers with about $14 \%$ relative to wildtype after five days in liquid simulated xylem medium (Fig. 3b). Conidiospore levels were restored in the HAC1-C strain. This suggests a further role for HAC1 function in subsequent steps of plant colonization, and this was investigated by tomato infection experiments. $\triangle H A C 1$ induced significantly less severe disease symptoms than wildtype 21 days after inoculation with conidiospores, resulting in approximately $90 \%$ healthy plants (Fig. $3 \mathrm{c}$ ). None of the $\triangle H A C 1$ inoculated plants showed heavy symptoms, and no hypocotyl discolorations were observed for any plant. In addition, no fungal outgrowth was observed for $\triangle H A C 1$ from stems of treated plants (Fig. 3c). The less virulent in planta phenotype of the $\triangle H A C 1$ strain was complemented by $H A C 1-C$ regarding induction of overall disease symptoms, discoloration of the hypocotyl and fungal outgrowth from stem sections. These results support the conclusion that a functional UPR regulator Hac1 is required for the initial colonization of the host root surface and sporulation as prerequisite for fungal propagation within the plant. Hac1 is therefore required for induction of severe disease symptoms in tomato.

\section{V. dahliae development and plant disease symptom induction require} pheromone response MAP kinase activities independently from the Ham5 scaffold

The unfolded protein response participates in cross regulation with the pheromone response MAPK pathway in the dimorphic plant pathogen $U$. maydis and this control prevents hypervirulence and maintains fungal biotrophy in the plant (Di Stasio et al., 2009; Heimel et al., 2013; Schmitz et al., 2019). Pheromone response MAPK pathways integrate the sensing of extracellular signals and activate downstream pathways that control pathogenic differentiation and fungal virulence for numerous fungi including V. dahliae (Jiang et al., 2018). Pathways are often assembled by scaffold proteins such as the yeast pheromone scaffold protein Ste5 to maintain specificity and prevent crosstalk between signalling pathways. The scaffold proteins Ham5/HamE of the 
pheromone response MAPK pathways of Neurospora crassa or Aspergillus nidulans are required for specific fungal developmental programs (Dettmann et al., 2014; Jonkers et al., 2014; Frawley et al., 2018). To date, corresponding scaffold proteins have not yet been analysed in plant pathogenic fungi and it is unknown whether they are required for pheromone response MAPK pathway mediated virulence. $V$. dahliae HAM5 was identified by reciprocal BLAST and the deduced protein shows $53 \%$ amino acid identity with the $N$. crassa scaffold homolog HAM-5. The $4906 \mathrm{bp}$ HAM5 pre-mRNA encodes a 1553 aa protein containing $\mathrm{N}$-terminal WD40 repeats and a coiled-coil domain at the C-terminus. The corresponding proteins of related filamentous fungi are similar in length and show conserved protein domains. $V$. dahliae MEK2 encodes a 522 aa MAP2K and VMK1 encodes a 355 aa MAPK, and both include ATP binding and serine/threonine-protein kinase active sites (Fig. 4).

The role of the scaffold Ham5 in the Vmk1 MAPK pathway for fungal development and virulence was compared to MEK2 and VMK1 through evaluation of the corresponding single and double deletion strains. The HAM5 deletion strain revealed wildtype-like growth and resting structure formation ex planta under stress and non-stress conditions, exemplified for CDM with sucrose or cellulose 9 days after inoculation (Fig. 5a). MEK2 and VMK1 single and double deletion strains with HAM5 exhibited a 10\% decrease in growth 9 days after inoculation, which was restored in the complementation strains MEK2-C and VMK1-C (Fig. 5a, b). In addition, $\triangle M E K 2$, $\triangle V M K 1, \triangle H A M 5 \triangle M E K 2$, and $\triangle H A M 5 \triangle V M K 1$ strains formed less microsclerotia on minimal medium with cellulose, and melanization of the colonies was reduced to less than half, whereas $\triangle H A M 5$ and $H A M 5-C$ strains displayed wildtype-like melanization. Phenotypes were restored by ectopic integration of the corresponding wildtype gene in MEK2-C and VMK1-C strains (Fig. 5a, b).

The impact of $V$. dahliae Ham5 was investigated in tomato plant infection experiments and compared to the core MAPK components Mek2 and Vmk1 (Fig. 5c). Symptoms of tomato plants inoculated with spores from $\triangle M E K 2, \triangle V M K 1, \triangle H A M 5 \triangle M E K 2$, or $\triangle H A M 5 \triangle V M K 1$ strains were comparable to mock plants after 21 days, and no hypocotyl discolorations were observed (Fig. 5c). The avirulent in planta phenotypes were complemented in experiments with VMK1-C and MEK2-C. Disease symptoms induced by the $\triangle H A M 5$ strain were indistinguishable from the wildtype control and plants displayed severe stunting and discoloration of the vascular tissue (Fig. 5c). 
These data corroborate that the requirement of the MAPK cascade components Vmk1 and Mek2 for virulence, growth and microsclerotia formation is independent of the presence of Ham5 and its isolation function as scaffold protein in $V$. dahliae. These results highlight the potential for crosstalk between UPR and this MAPK module signalling pathway, given that both promote fungal virulence, growth and microsclerotia formation.

\section{The $V$. dahliae oleate $\Delta 12$-fatty acid desaturase Ode1 promotes fungal differentiation with only a minor impact on virulence}

Activation of the yeast pheromone response MAPK pathway relies on the perception of peptide pheromones (Bardwell, 2005). To coordinate their development, several filamentous fungi produce oxylipins instead of pheromones as signalling molecules, which are as well connected to host-fungus communication (Calvo et al., 2001; Brodhagen et al., 2008; Brodhun et al., 2009; Reverberi et al., 2010; Scala et al., 2014; Fischer \& Keller, 2016; Patkar \& Naqvi, 2017). Secretion of these lipid metabolites potentially relies on the UPR control of the capacity and quality of ER mediated secretion. We examined functions of $V$. dahliae oxylipins as potential signals for development and virulence, and focused an oleate $\Delta 12$-fatty acid desaturase catalysing the oxidation of oleic acid to linoleic acid, the major precursor of fungal oxylipins. The impact of the oleate $\Delta 12$-fatty acid desaturase OdeA on differentiation was described in different Aspergilli, but has not yet been examined in plant pathogens (Calvo et al., 2001; Chang et al., 2004; Wilson et al., 2004).

$V$. dahliae ODE1 was identified as the homolog to $A$. nidulans odeA by reciprocal BLAST search of the amino acid sequences with $66 \%$ aa sequence identity of the deduced proteins. The Ode1 protein with a length of 481 aa and a predicted molecular weight of $54 \mathrm{kDa}$ contains two fatty acid desaturase (FAD) domains (Fig. 6a). The cytosolic catalytic centre of $\Delta 12$-fatty acid desaturases is formed by conserved $\mathrm{N}$ - and C-terminal histidine clusters described as FAD domains and iron atoms provided by the membrane-bound donor cytochrome b5 (Los \& Murata, 1998). V. dahliae Ode1 was predicted as a transmembrane protein with four hydrophobic transmembrane helices, two short non-cytosolic regions, and three hydrophilic cytosolic regions, including the $\mathrm{N}$ - and C-termini of the protein (Fig. 6b).

V. dahliae ODE1 deletion and complementation strains harbouring ODE1 C-terminally fused to GFP at the endogenous locus under control of the native promoter and 
terminator were constructed. The predicted molecular weight for Ode1 fused to GFP with $81 \mathrm{kDa}$ was confirmed by immunoblots (Fig. 6c). Fluorescence microscopy of young hyphae revealed that the Ode1-GFP protein is primarily localized to ER membranes surrounding the nucleus and to a minor extend to plasma membranes and close to tips of growing hyphae resembling perinuclear and cortical ER structures (Fig. $6 \mathrm{~d}-\mathrm{f})$.

The impact of the $V$. dahliae oleate $\Delta 12$-fatty acid desaturase Ode1 on fungal growth and differentiation was examined by comparing the ODE1 deletion to the wildtype strain under different physiological and membrane stress inducing conditions. Vegetative growth of the $\triangle O D E 1$ strain was significantly decreased with or without stressors (Fig. 7a). The ODE1 deletion strain displayed the most severe decrease in vegetative growth on medium containing cellulose, with a $\sim 50 \%$ reduction of the colony diameter after 9 days. This defect was complemented by ODE1-GFP (Fig. 7a, b). The observed growth defect of $\triangle O D E 1$ was partially compensated by supplementation of media with linoleic acid, resulting in a relative colony diameter of about $70 \%$ (Fig. $7 \mathrm{~b}$ ). Reduced melanization correlated with the decrease in growth of colonies formed by $\triangle O D E 1$ on cellulose medium. Colony cross sections and microscopy of fungal material from colony centres revealed the formation of wildtype-like microsclerotia with regard to size, shape and melanization by the $\triangle O D E 1$ strain (Fig. 7a).

Tomato plant infection experiments addressed the impact of $O D E 1$ on the virulence of $V$. dahliae. Tomato plants inoculated with $\triangle O D E 1$ spores showed only slightly reduced numbers of plants with disease symptoms after 21 days compared to stunting and hypocotyl discolorations of wildtype infected plants (Fig. 7c). This suggests a strong contribution of the single gene $O D E 1$ to fungal growth but only a minor contribution to the virulence of $V$. dahliae.

These data hint at a complex interplay between biosynthesis of linoleic acids as precursors of oxylipins, the secretion capacity moderating UPR, and the scaffoldindependent pheromone response MAPK pathway, which perceives external signals and activates downstream pathways. This interplay results in promotion or reduction of fungal growth and influences the outcome of the interaction of $V$. dahliae with its host plant. 


\section{Discussion}

The unfolded protein response and the Ham5 scaffold-independent pheromone response MAPK pathway form important distinct signalling hubs for $V$. dahliae plant pathogenicity. The UPR regulator Hac1 is essential for induction of resting structure formation and conidiation, the first contact with the plant roots and propagation within the plant. The pheromone response MAPK pathway does not require the scaffold Ham5 to support maturation and melanization of microsclerotia as well as prompting disease symptoms as severe stunting and discoloration of the vascular tissue. The linoleic acid synthesizing oleate $\Delta 12$-fatty acid desaturase Ode1 primarily affects fungal growth with only a small contribution to virulence of $V$. dahliae. The interplay between these signalling pathway components in $V$. dahliae growth, development and induction of plant disease is summarized in Fig. 8.

The V. dahliae UPR regulator Hac1 has conserved, as well as species-specific impacts on fungal differentiation and is important for virulence. The $V$. dahliae HAC1 mRNA contains an unconventional intron with sequence and structural similarity to Ire1 spliced introns in other organisms. In contrast to wildtype-like HAC1 gene expression levels in the complementation strain harbouring the entire $H A C 1$ gene, $H A C 1^{i}-H A$ and $H A C 1 u_{-} H A$ strains harbouring either one of two HAC1 splice variants without conventional intron in its genome displayed decreased expression. This might result from intron-mediated enhanced transcription of HAC1.

Only the Hac1 protein resulting from translation of unconventionally spliced mRNA, but not from the uninduced variant, produced sufficient protein amounts for detection by immunoblots. Translation of HAC1u mRNA might be blocked or result in an unstable protein due to similar mechanisms as described for other ascomycetes. In S. cerevisiae base-pairing interaction between unconventional intron and 5'UTR leads to inhibition of ribosomal translation (Chapman \& Walter, 1997; Rüegsegger et al., 2001). Additionally, accelerated degradation of Hac1 ${ }^{\mathrm{u}}$ proteins was described in yeast (Di Santo et al., 2016). Due to shortened intron length in HAC1u mRNA of filamentous ascomycetes, a similar mechanism for translation inhibition as in yeast is not possible (Mulder \& Nikolaev, 2009). Rather, an impact of upstream ORFs on translational repression of unspliced HAC1 mRNA was assumed (Saloheimo et al., 2003; Mulder et al., 2004), but studies in Aspergillus niger revealed base-pairing of the 5'UTR of HAC1 mRNA with an inverted repeat sequence as translation attenuation mechanism (Mulder \& Nikolaev, 2009). Truncation of the $5^{\prime}$ UTR was described to foster translation of HAC1 
mRNA upon ER stress in different Aspergilli, T. reesei and A. brassisicola (Saloheimo et al., 2003; Mulder et al., 2004; Joubert et al., 2011).

$V$. dahliae is viable in the presence of constitutively induced HAC1 mRNA and does not require the presence of the uninduced variant. In contrast, regulatory roles of the unspliced mRNA homolog and the deduced protein were proposed for U. maydis (Heimel et al., 2013). Here, overexpression of the induced mRNA resulted in UPR hyperactivation and lethality unless unspliced mRNA was present in the cell (Heimel et al., 2013).

In S. cerevisiae, U. maydis and C. neoformans the UPR is not required for vegetative growth and sporulation in the absence of ER stress (Nikawa et al., 1996; Kaufman, 1999; Cheon et al., 2011; Heimel et al., 2013). In contrast, deletion of HAC1 or genomic integration of the unconventionally spliced HAC1 mRNA variant affects growth and conidiation without stress in different filamentous ascomycetes including plant pathogens (Mulder \& Nikolaev, 2009; Joubert et al., 2011; Carvalho et al., 2012; Tang et al., 2015). A basal UPR activity under non-stress conditions has been observed in several fungi (Heimel, 2015). Constitutive activation of the UPR might alter the control of genes involved in growth and developmental processes (Heimel, 2015). The $V$. dahliae $H A C 1$ deletion strain was also generally impaired in growth and was not additionally impaired in response to tunicamycin. Expression of the induced HAC1 splice variant improved the ability to cope with ER stress, although expression levels of the induced splice variant were lower compared to wildtype. Under non-stress conditions, HAC1i-HA displayed reduced growth, which might be explained by differential regulation of genes supporting growth in correlation to a hyperactive UPR. This idea is supported by the finding, that the induced mRNA variant of the UPR regulator could be amplified from cultures grown under non-stress conditions in V. dahliae.

HAC1 of the vascular pathogen $V$. dahliae is not only required for growth and conidiation in the absence of typical ER stress inducing conditions, but is also essential for the formation of microsclerotia. HAC1 deletion strains do not form these resting structures under any tested condition. Decreased expression of the ectopically integrated $H A C 1$ gene lacking the conventional intron in the deletion background was sufficient to complement the microsclerotia phenotype in HAC14-HA. For HAC1i-HA increased microsclerotia formation was observed. One possible explanation for HAC1 being essential for microsclerotia production is that the UPR is a checkpoint to induce 
resting structure formation after sensing of unfavourable conditions. The mechanisms activating this process in $V$. dahliae are not yet understood, even if several candidates were shown to influence microsclerotia production such as the pheromone response MAPK pathway components Vmk1 and Mek2. An essential protein for microsclerotia formation is the transcription factor Som1 (Bui et al., 2019). Som1 is involved in regulation of a subset of genes, such as the Verticillium transcription activators of adhesion Vta2 and Vta3, which are involved in adhesion and microsclerotia formation (Tran et al., 2014; Bui et al., 2019). In S. cerevisiae Hac1 is involved in regulation of flocculin genes and interacts with the general control of amino acid biosynthesis (Herzog et al., 2013), and ER stressed cells display increased flocculation (Scrimale et al., 2009). The absence of microsclerotia in Som1 and Hac1-deficient V. dahliae strains hints to a crosstalk between Som1 and the UPR during regulation of microsclerotia formation.

In U. maydis the Hac1 orthologue is required to induce biotrophic growth in planta (Heimel et al., 2010). In contrast, UPR components are involved in initial penetration of the plant surface by M. oryzae (Yi et al., 2009; Tang et al., 2015; Jiang et al., 2018). The $V$. dahliae UPR regulator Hac1 has a major impact on the fungal ability to colonize host plants, and HAC1 is required for efficient colonization of the root surface as first step. The subsequent penetration and initial root cortex invasion does not require Hac1, which is reminiscent of the finding that initial penetration of the plant surface was unaffected in the appressorium-forming fungus $A$. brassicicola as well as in $U$. maydis (Heimel et al., 2010; Joubert et al., 2011).

Tomato plants treated with the V. dahliae HAC1 deletion strain displayed severely decreased disease symptoms and fungal re-isolation from treated stems was not possible. Successful propagation within the host requires fungal spreading within the vascular system by conidiation, which is defective in the HAC1 deletion strain. In addition, the UPR considerably contributes to the necessary adaptation of secretory capacities during host colonization of pathogens and the processing and secretion of fungal effectors in U. maydis (Richie et al., 2011; Heimel et al., 2013; Hampel et al., 2016; Pinter et al., 2019).

Similar to the UPR, the pheromone response MAPK pathway is required for virulence of $V$. dahliae and contributes to growth and microsclerotia formation. Different MAPK pathways can share components, like MAP kinases, adaptor proteins or upstream kinases. For certain cascades scaffold proteins are necessary to bring components in 
proximity and maintain pathway specificity by isolation (Schaeffer \& Weber, 1999; Patterson et al., 2010). Scaffold proteins are described for the pheromone response MAPK pathway in different filamentous ascomycetes and support fusion of fungal cells (Dettmann et al., 2014; Jonkers et al., 2014; Frawley et al., 2018). The N. crassa scaffold protein HAM-5 assembles the MAPK cascade during chemotropic growth and positively influences growth and differentiation (Li et al., 2005; Aldabbous et al., 2010; Dettmann et al., 2014; Jonkers et al., 2014). The homologous A. nidulans scaffold protein HamE is required for sexual and asexual development and secondary metabolite production (Frawley et al., 2018). The role of homologous scaffold proteins was yet unstudied in plant pathogens. Our findings demonstrate that pheromone response MAPK cascade mediated growth, differentiation and virulence are independent from isolation of this pathway by the Ham5 scaffold protein in $V$. dahliae.

In the basidiomycete

U. maydis the phosphatase Rok1 (regulator of Kpp2) is responsible for dephosphorylation of the partially redundant MAP kinases Kpp2 and Kpp6 (kinase PCR-product 2/6) (Di Stasio et al., 2009). U. maydis rok1 deficient mutants displayed a hypervirulent phenotype on maize plants. A scaffold protein involved in isolation of the MAPK cascade was not described in $U$. maydis. Recently it was shown, that Rok1 activity is regulated and induced by the UPR pathway (Schmitz et al., 2019). The orthologous phosphatase in V.dahliae (VDAG_JR2_Chr7g08960a) with 803 aa harbours a dual specificity phosphatase domain (337-528 aa, IPR020422) and shows high similarity to corresponding proteins of filamentous ascomycetes, but is more distantly related to $U$. maydis Rok1 or S. cerevisiae Msg5 (Supplementary Figure S4). As in the basidiomycete $U$. maydis the corresponding protein of the filamentous ascomycete $M$. oryzae functions in dephosphorylation of the pheromone responsive MAPK (Wang et al., 2017). This observation suggests that future studies should investigate the possibility of a connection between UPR and pheromone response MAPK in the vascular ascomycete pathogen $V$. dahliae.

Biosynthesis and secretion of lipid metabolites involved in growth, differentiation and virulence of pathogenic fungi might be controlled by the pheromone response MAPK cascade and the ER homeostasis moderating UPR. In several cases, the virulence of plant pathogenic fungi depends on oxylipin signalling (Brodhun \& Feussner, 2011). Characterization of the $V$. dahliae oleate $\Delta 12$-fatty acid desaturase Ode1, catalysing the biosynthesis of linoleic acid, revealed an important contribution to fungal growth 
with only minor impact on induction of disease symptoms. Similarly, a general decrease in growth was observed for A. nidulans and Aspergillus parasiticus upon deletion of homologous oleate $\Delta 12$-fatty acid desaturases (Calvo et al., 2001; Chang et al., 2004; Wilson et al., 2004). V. dahliae ODE1 is dispensable for formation of wildtype-like resting structures, whereas deletion of the $A$. parasiticus ODE1 homolog resulted in loss of sclerotia development (Chang et al., 2004; Wilson et al., 2004).

Despite its impact on growth, $V$. dahliae Ode1 is not required for induction of severe disease symptoms in tomato plants. It is possible, that different oleate $\Delta 12$-fatty acid desaturases encoded in the $V$. dahliae genome are responsible for linoleic acid biosynthesis during plant colonization and compensate for loss of Ode1 function. In addition, the $O D E 1$ deletion strain might be able to use plant-derived unsaturated fatty acids to compensate for the growth defect. Plant linoleic acid and the derived oxylipins are recognized as mimics of fungal signalling molecules and promote sporulation and mycotoxin production in Aspergilli (Burow et al., 1997; Calvo et al., 1999; Wilson et al., 2004; Brodhagen et al., 2008; Gao \& Kolomiets, 2009; Horowitz Brown et al., 2009; Reverberi et al., 2010).

In conclusion, V. dahliae Hac1 regulated unfolded protein response and a scaffoldindependent pheromone response MAPK pathway are important for both resting structure formation and plant infection. They are potentially interconnected and represent interesting targets to control growth, survival of microsclerotia in the soil and propagation in the plant as a means to reduce the impact on crops of this important vascular pathogen.

\section{Acknowledgments}

The authors thank N. Scheiter for technical assistance, K. Heimel for fruitful discussions and carefully reading the manuscript, S. Balnojan for construction of the plasmid pME4815, J. Teer for construction of the plasmid pME4976, A. Nagel for construction of the VGB493/VGB494, A. Nagel, J. Teer and E. F. Hettwer for support as student research assistants, as well as $M$. Leonard and A. Höfer for support. This work was funded by the Deutsche Forschungsgemeinschaft (DFG) IRTG 2172 (JS, IM), an NSERC CREATE award (JWK) and DFG BR1502/15-1 (RH, GHB). 


\section{Author contributions}

Conceived and designed the experiment JS, RH, IM, JWK and GHB. Performed the experiments JS, RH, IM, and RB. Analysed the data JS, RH, GHB. Wrote the paper $\mathrm{JS}, \mathrm{RH}$, and $\mathrm{GHB}$.

\section{References}

Aldabbous MS, Roca MG, Stout A, Huang I-C, Read ND, Free SJ. 2010. The ham$5, \mathrm{rcm}-1$ and $\mathrm{rco}-1$ genes regulate hyphal fusion in Neurospora crassa. Microbiology. doi: 10.1099/mic.0.040147-0.

Almagro Armenteros JJ, Sønderby CK, Sønderby SK, Nielsen H, Winther O. 2017. DeepLoc: prediction of protein subcellular localization using deep learning. Bioinformatics 33: 3387-3395.

Alvaro CG, Thorner J. 2016. Heterotrimeric G protein-coupled receptor signaling in yeast mating pheromone response. Journal of Biological Chemistry. doi. 10.1074/jbc.R116.714980.

Bardwell L. 2005. A walk-through of the yeast mating pheromone response pathway. Peptides. doi: 10.1016/j.peptides.2004.10.002.

Brodhagen M, Tsitsigiannis DI, Hornung E, Goebel C, Feussner I, Keller NP. 2008. Reciprocal oxylipin-mediated cross-talk in the Aspergillus-seed pathosystem. Molecular Microbiology. doi: 10.1111/j.1365-2958.2007.06045.x.

Brodhun F, Feussner I. 2011. Oxylipins in fungi. FEBS Journal: doi: 10.1111/j.17424658.2011.08027.x.

Brodhun F, Go C, Hornung E, Feussner I. 2009. Identification of PpoA from Aspergillus nidulans as a fusion protein of a fatty acid heme dioxygenase/ peroxidase and a cytochrome P450. The Journal of biological chemistry. doi: 10.1074/jbc.M809152200.

Bui T, Harting R, Braus- Stromeyer SA, Tran V, Leonard M, Höfer A, Abelmann A, Bakti F, Valerius O, Schlüter R, et al. 2019. Verticillium dahliae transcription factors Som1 and Vta3 control microsclerotia formation and sequential steps of plant root penetration and colonisation to induce disease. New Phytologist. doi: 10.1111/nph.15514.

Burow GB, Nesbitt TC, Dunlap J, Keller NP. 1997. Seed lipoxygenase products modulate Aspergillus mycotoxin biosynthesis. Molecular Plant-Microbe Interactions. doi: 10.1094/MPMI.1997.10.3.380.

Calvo AM, Gardner HW, Keller NP. 2001. Genetic connection between fatty acid metabolism and sporulation in Aspergillus nidulans. doi: 10.1074/jbc.M100732200.

Calvo AM, Hinze LL, Gardner HW, Keller NP. 1999. Sporogenic effect of polyunsaturated fatty acids on development of Aspergillus spp. Applied and Environmental Microbiology 65: 3668-3673.

Carvalho NDSP, Jørgensen TR, Arentshorst M, Nitsche BM, van den Hondel CA, 
Archer DB, Ram AFJ. 2012. Genome-wide expression analysis upon constitutive activation of the HacA bZIP transcription factor in Aspergillus niger reveals a coordinated cellular response to counteract ER stress. BMC genomics. doi: 10.1186/1471-2164-13-350.

Chang P, Wilson RA, Keller NP, Cleveland TE. 2004. Deletion of the $\Delta 12$-oleic acid desaturase gene of a nonaflatoxigenic Aspergillus parasiticus field isolate affects conidiation and sclerotial development. Journal of Applied Microbiology. doi: 10.1111/j.1365-2672.2004.02424.x.

Chapman RE, Walter P. 1997. Translational attenuation mediated by an mRNA intron. Current biology, CB 7: 850-859.

Cheon SA, Jung KW, Chen YL, Heitman J, Bahn YS, Kang HA. 2011. Unique evolution of the UPR pathway with a novel bZIP transcription factor, HxL1, for controlling pathogenicity of Cryptococcus neoformans. PLOS Pathogens. doi: 10.1371/journal.ppat.1002177.

Conn PM. 2011. The unfolded protein response and cellular stress. In: Conn MP, ed. Methods in Enzymology. Los Angeles, CA, USA: Elsevier.

Cox JS, Shamu CE, Walter P. 1993. Transcriptional induction of genes encoding endoplasmic reticulum resident proteins requires a transmembrane protein kinase. Cell. doi: 10.1016/0092-8674(93)90648-A.

Cullen PJ, Sprague GF, Jr. 2012. The regulation of filamentous growth in yeast. Genetics. doi: 10.1534/genetics.111.127456.

Dettmann A, Heilig Y, Valerius O, Ludwig S, Seiler S. 2014. Fungal communication requires the MAK-2 pathway elements STE-20 and RAS-2, the NRC-1 adapter STE50 and the MAP kinase scaffold HAM-5. PLOS Genetics. doi: 10.1371/journal.pgen.1004762.

Edgar RC. 2004. MUSCLE: multiple sequence alignment with high accuracy and high throughput. Nucleic acids research. doi: 10.1093/nar/gkh340.

Eynck C, Koopmann B, Grunewaldt-Stoecker G, Karlovsky P, von Tiedemann A. 2007. Differential interactions of Verticillium longisporum and $V$. dahliae with Brassica napus detected with molecular and histological techniques. European Journal of Plant Pathology. doi: 10.1007/s10658-007-9144-6.

Fischer GJ, Keller NP. 2016. Production of cross-kingdom oxylipins by pathogenic fungi: An update on their role in development and pathogenicity. Journal of microbiology. doi: 10.1007/s12275-016-5620-z.

Fitzell R, Evans G, Fahy P. 1980. Studies on the colonization of plant roots by Verticillium dahliae Klebahn with use of immunofluorescent staining. Australian Journal of Botany 28: doi: 10.1071/BT9800357.

Fradin EF, Thomma BPHJ. 2006. Physiology and molecular aspects of Verticillium wilt diseases caused by $V$. dahliae and $V$. albo-atrum. Molecular plant pathology. doi: 10.1111/j.1364-3703.2006.00323.x.

Fradin EF, Zhang Z, Juarez Ayala JC, Castroverde CDM, Nazar RN, Robb J, Liu C-M, Thomma BPHJ. 2009. Genetic dissection of Verticillium wilt resistance mediated by tomato Ve1. Plant physiology 150: 320-332. 
Frawley D, Karahoda B, Sarikaya Bayram Ö, Bayram Ö. 2018. The HamE scaffold positively regulates MpkB phosphorylation to promote development and secondary metabolism in Aspergillus nidulans. Scientific Reports. doi: 10.1038/s41598-01834895-6.

Gao X, Kolomiets M V. 2009. Host-derived lipids and oxylipins are crucial signals in modulating mycotoxin production by fungi. Toxin Reviews. doi: 10.1080/15569540802420584.

Geer LY, Marchler-Bauer A, Geer RC, Han L, He J, He S, Liu C, Shi W, Bryant SH. 2010. The NCBI BioSystems database. Nucleic Acids Research. doi: 10.1093/nar/gkp858.

Gonzalez TN, Sidrauski C, Dörfler S, Walter P. 1999. Mechanism of nonspliceosomal mRNA splicing in the unfolded protein response pathway. The EMBO journal. doi: 10.1093/emboj/18.11.3119.

Griffiths DA. 1970. The fine structure of developing microsclerotia of Verticillium dahliae Kleb. Archiv für Mikrobiologie. doi: 10.1007/BF00408881.

Guillemette T, Calmes B, Simoneau P, Index FH. 2014. Impact of the UPR on the virulence of the plant fungal pathogen $A$. brassicicola. Virulence. doi: $10.4161 /$ viru.26772.

Hampel M, Jakobi M, Schmitz L, Meyer U, Finkernagel F, Doehlemann G, Heimel K. 2016. Unfolded protein response (UPR) regulator Cib1 controls expression of genes encoding secreted virulence factors in Ustilago maydis. PLOS ONE. doi: 10.1371/journal.pone.0153861.

Heimel K. 2015. Unfolded protein response in filamentous fungi - implications in biotechnology. Applied Microbiology and Biotechnology. doi: 10.1007/s00253-0146192-7.

Heimel K, Freitag J, Hampel M, Ast J. 2013. Crosstalk between the unfolded protein response and pathways that regulate pathogenic development in Ustilago maydis. The Plant Cell. doi: 10.1105/tpc.113.115899.

Heimel K, Scherer M, Schuler D, Kämper J. 2010. The Ustilago maydis Clp1 protein orchestrates pheromone and b-dependent signaling pathways to coordinate the cell cycle and pathogenic development. The Plant cell: doi: 10.1105/tpc.110.076265.

Herzog B, Popova B, Jakobshagen A, Shahpasandzadeh H, Braus GH. 2013. Mutual cross talk between the regulators Hac1 of the unfolded protein response and Gcn4 of the general amino acid control of Saccharomyces cerevisiae. Eukaryotic cell. doi: 10.1128/EC.00123-13.

Hetz C. 2012. The unfolded protein response: controlling cell fate decisions under ER stress and beyond. Nature Reviews Molecular Cell Biology. doi: 10.1038/nrm3270.

Hooks KB, Griffiths-Jones S. 2011a. Conserved RNA structures in the non-canonical Hac1/Xbp1 intron. RNA biology. doi: 10.4161/rna.8.4.15396.

Hooks KB, Griffiths-Jones S. 2011b. Conserved RNA structures in the non-canonical Hac1/Xbp1 intron. RNA biology 8: 552-556.

Horowitz Brown S, Scott JB, Bhaheetharan J, Sharpee WC, Milde L, Wilson RA, Keller NP. 2009. Oxygenase coordination is required for morphological transition and 
the host-fungus interaction of Aspergillus flavus. Molecular Plant-Microbe Interactions. doi: 10.1094/MPMI-22-7-0882.

Jiang C, Zhang X, Liu H, Xu J-R. 2018. Mitogen-activated protein kinase signaling in plant pathogenic fungi. PLOS Pathogens. doi: 10.1371/journal.ppat.1006875.

Jones P, Binns D, Chang H-Y, Fraser M, Li W, McAnulla C, McWilliam H, Maslen J, Mitchell A, Nuka G, et al. 2014. InterProScan 5: genome-scale protein function classification. Bioinformatics: doi: 10.1093/bioinformatics/btu031.

Jonkers W, Leeder AC, Ansong C, Wang Y, Yang F, Starr TL, Camp DG, Smith RD, Glass NL. 2014. HAM-5 functions as a MAP kinase scaffold during cell fusion in Neurospora crassa. PLoS Genetics. doi: 10.1371/journal.pgen.1004783.

Joubert A, Simoneau P, Campion C, Poupard P, François JM, Georgeault S, Sellier E, Guillemette T. 2011. Impact of the unfolded protein response on the pathogenicity of the necrotrophic fungus Alternaria brassicicola. Molecular Microbiology. doi: 10.1111/j.1365-2958.2010.07522.x.

Kaufman RJ. 1999. Stress signaling from the lumen of the endoplasmic reticulum: 13: $1211-1233$.

Kersey PJ, Allen JE, Allot A, Barba M, Boddu S, Bolt BJ, Carvalho-Silva D, Christensen M, Davis P, Grabmueller C, et al. 2018. Ensembl Genomes 2018: an integrated omics infrastructure for non-vertebrate species. Nucleic Acids Research. doi: $10.1093 /$ nar/gkx1011.

Kimmig P, Diaz M, Zheng J, Williams CC, Lang A, Aragón T, Li H, Walter P. 2012. maintain protein homeostasis. eLife. doi: 10.7554/eLife.00048.

Klosterman SJ, Atallah ZK, Vallad GE, Subbarao K V. 2009. Diversity, pathogenicity, and management of Verticillium species. Annual Review of Phytopathology. doi: 10.1146/annurev-phyto-080508-081748.

Kohno K, Normington K, Sambrook J, Gething MJ, Mori K. 1993. The promoter region of the yeast KAR2 $(\mathrm{BiP})$ gene contains a regulatory domain that responds to the presence of unfolded proteins in the endoplasmic reticulum. Molecular and cellular biology. doi: 10.1128/mcb.13.2.877.

Kosugi S, Hasebe M, Tomita M, Yanagawa H. 2009. Systematic identification of cell cycle-dependent yeast nucleocytoplasmic shuttling proteins by prediction of composite motifs. Proceedings of the National Academy of Sciences. doi: 10.1073/pnas.0900604106.

Kozutsumi Y, Segal M, Normington K, Gething M-J, Sambrook J. 1988. The presence of malfolded proteins in the endoplasmic reticulum signals the induction of glucose-regulated proteins. Nature. doi: 10.1038/332462a0.

Krishnan K, Askew DS. 2014. The fungal UPR: a regulatory hub for virulence traits in the mold pathogen Aspergillus fumigatus. Virulence. doi: 10.4161/viru.26571.

Li D, Bobrowicz P, Wilkinson HH, Ebbole DJ. 2005. A mitogen-activated protein kinase pathway essential for mating and contributing to vegetative growth in Neurospora crassa. Genetics. doi: 10.1534/genetics.104.036772. 
Li J-J, Zhou L, Yin C-M, Zhang D-D, Klosterman SJ, Wang B-L, Song J, Wang D, Hu X-P, Subbarao K V., et al. 2019. The Verticillium dahliae Sho1-MAPK pathway regulated melanin biosynthesis is required for cotton infection. Environmental Microbiology 21: 4852-4874.

Los DA, Murata N. 1998. Structure and expression of fatty acid desaturases. Biochimica et Biophysica Acta - Lipids and Lipid Metabolism 1394: 3-15.

Luo X, Xie C, Dong J, Yang X, Sui A. 2014. Interactions between Verticillium dahliae and its host: vegetative growth, pathogenicity, plant immunity. Applied microbiology and biotechnology. doi: 10.1007/s00253-014-5863-8.

Miyazaki T, Nakayama H, Nagayoshi Y, Kakeya H, Kohno S. 2013. Dissection of glabrata. PLoS Pathogens. doi: 10.1371/journal.ppat.1003160.

Mori K, Kawahara T, Yoshida H, Yanagi H, Yura T. 1996. Signalling from endoplasmic reticulum to nucleus: transcription factor with a basic-leucine zipper motif is required for the unfolded protein-response pathway. Genes to Cells. doi: 10.1046/j.1365-2443.1996.d01-274.x.

Mori K, Ma W, Gething MJ, Sambrook J. 1993. A transmembrane protein with a cdc2+/CDC28-related kinase activity is required for signaling from the ER to the nucleus. Cell. doi: 10.1016/0092-8674(93)90521-q.

Mori K, Ogawa N, Kawahara T, Yanagi H, Yura T. 1998. Palindrome with spacer of one nucleotide is characteristic of the cis -acting unfolded protein response element in Saccharomyces cerevisiae. Journal of Biological Chemistry. doi: 10.1074/jbc.273.16.9912.

Mulder HJ, Nikolaev I. 2009. HacA-dependent transcriptional switch releases hacA mRNA from a translational block upon endoplasmic reticulum stress. Eukaryotic cell. doi: 10.1128/EC.00131-08.

Mulder HJ, Saloheimo M, Penttilä M, Madrid SM. 2004. The transcription factor HACA mediates the unfolded protein response in Aspergillus niger, and up-regulates its own transcription. Molecular Genetics and Genomics. doi: 10.1007/s00438-0030965-5.

Nikawa J, Akiyoshi M, Hirata S, Fukuda T. 1996. Saccharomyces cerevisiae IRE2/HAC1 is involved in IRE1-mediated KAR2 expression. Nucleic acids research. doi: 10.1093/nar/24.21.4222.

Okamura K, Kimata Y, Higashio H, Tsuru A, Kohno K. 2000. Dissociation of Kar2p/BiP from an ER sensory molecule, Ire1p, triggers the unfolded protein response in yeast. Biochemical and biophysical research communications. doi: 10.1006/bbrc.2000.3987.

Patkar RN, Naqvi NI. 2017. Fungal manipulation of hormone-regulated plant defense. PLOS Pathogens. doi: 10.1371/journal.ppat.1006334.

Patterson JC, Klimenko ES, Thorner J. 2010. Single-cell analysis reveals that insulation maintains signaling specificity between two yeast MAPK pathways with common components. Science signaling. doi: 10.1161/ATVBAHA.114.303112.ApoAI. 
Pegg GF, Brady BL. 2002. Verticillium Wilts. Wallingford: CABI Pub.

Pinter N, Hach CA, Hampel M, Rekhter D, Zienkiewicz K, Feussner I, Poehlein A, Daniel R, Finkernagel F, Heimel K. 2019. Signal peptide peptidase activity connects the unfolded protein response to plant defense suppression by Ustilago maydis. PLOS Pathogens. doi: 10.1371/journal.ppat.1007734.

Qi X, Zhou S, Shang X, Wang X. 2016. VdSho1 regulates growth, oxidant adaptation and virulence in Verticillium dahliae. Journal of Phytopathology. doi: 10.1111/jph.12527.

Rauyaree P, Ospina-Giraldo MD, Kang S, Bhat RG, Subbarao KV, Grant SJ, Dobinson KF. 2005. Mutations in VMK1, a mitogen-activated protein kinase gene, affect microsclerotia formation and pathogenicity in Verticillium dahliae. Current Genetics. doi: 10.1007/s00294-005-0586-0.

Reverberi M, Punelli F, Scarpari M, Camera E, Zjalic S, Ricelli A, Fanelli C, Fabbri AA. 2010. Lipoperoxidation affects ochratoxin A biosynthesis in Aspergillus ochraceus and its interaction with wheat seeds. Applied Microbiology and Biotechnology. doi: 10.1007/s00253-009-2220-4.

Richie DL, Feng X, Hartl L, Aimanianda V, Krishnan K, Powers-Fletcher M V, Watson DS, Galande AK, White SM, Willett T, et al. 2011. The virulence of the opportunistic fungal pathogen Aspergillus fumigatus requires cooperation between the endoplasmic reticulum-associated degradation pathway (ERAD) and the unfolded protein response (UPR). Virulence. doi: 10.4161/VIRU.2.1.13345.

Rüegsegger U, Leber JH, Walter P. 2001. Block of HAC1 mRNA translation by longrange base pairing is released by cytoplasmic splicing upon induction of the unfolded protein response. Cell. doi: 10.1016/s0092-8674(01)00505-0.

Saloheimo M, Valkonen M, Penttilä M. 2003. Activation mechanisms of the HACImediated unfolded protein response in filamentous fungi. Molecular Microbiology 47: 1149-1161.

Di Santo R, Aboulhouda S, Weinberg DE. 2016. The fail-safe mechanism of posttranscriptional silencing of unspliced HAC1 mRNA. eLife. doi: 10.7554/eLife.20069.

Sarmiento-Villamil JL, Prieto P, Klosterman SJ, García-Pedrajas MD. 2018. Characterization of two homeodomain transcription factors with critical but distinct roles in virulence in the vascular pathogen Verticillium dahliae. Molecular Plant Pathology. doi: 10.1111/mpp.12584.

Scala V, Giorni P, Cirlini M, Ludovici M, Visentin I, Cardinale F, Fabbri AA, Fanelli C, Reverberi M, Battilani P, et al. 2014. LDS1-produced oxylipins are negative regulators of growth, conidiation and fumonisin synthesis in the fungal maize pathogen Fusarium verticillioides. Frontiers in microbiology. doi: 10.3389/fmicb.2014.00669.

Schaeffer HJ, Weber MJ. 1999. Mitogen-activated protein kinases: specific messages from ubiquitous messengers. Molecular and cellular biology. doi: 10.1128/mcb.19.4.2435.

Schmitz L, Schwier MA, Heimel K. 2019. The unfolded protein response regulates pathogenic development of Ustilago maydis by Rok1-dependent inhibition of matingtype signaling. mBio. doi: 10.1128/mBio.02756-19. 
Scrimale T, Didone L, Bentley KL de M, Krysan DJ. 2009. The unfolded protein response is induced by the cell wall integrity mitogen-activated protein kinase signaling cascade and is required for cell wall integrity in Saccharomyces cerevisiae. Molecular Biology of the Cell. doi: 10.1091/MBC.E08-08-0809.

Sidrauski C, Walter P. 1997. The transmembrane kinase Ire1p is a site-specific endonuclease that initiates mRNA splicing in the unfolded protein response. Cell. doi: 10.1016/S0092-8674(00)80369-4.

Di Stasio M, Brefort T, Mendoza-Mendoza A, Münch K, Kahmann R. 2009. The dual specificity phosphatase Rok1 negatively regulates mating and pathogenicity in Ustilago maydis. Molecular Microbiology. doi: 10.1111/j.1365-2958.2009.06747.x.

Su X, Lu G, Rehman L, Li X, Sun L, Guo H, Cheng H. 2018. mCherry-labeled Verticillium dahliae could be utilized to investigate its pathogenicity process in Nicotiana benthamiana. Genes. doi: 10.3390/genes9100508.

Tamura K, Stecher G, Peterson D, Filipski A, Kumar S. 2013. MEGA6: Molecular Evolutionary Genetics Analysis version 6.0. Molecular biology and evolution. doi: 10.1093/molbev/mst197.

Tang W, Ru Y, Hong L, Zhu Q, Zuo R, Guo X, Wang J, Zhang H, Zheng X, Wang $\mathbf{P}$, et al. 2015. System-wide characterization of bZIP transcription factor proteins involved in infection-related morphogenesis of Magnaporthe oryzae. Environmental microbiology. doi: 10.1111/1462-2920.12618.

Thompson JD, Higgins DG, Gibson TJ. 1994. CLUSTAL W: improving the sensitivity of progressive multiple sequence alignment through sequence weighting, positionspecific gap penalties and weight matrix choice. Nucleic acids research. doi: 10.1093/nar/22.22.4673.

Tran VT, Braus-Stromeyer SA, Kusch H, Reusche M, Kaever A, Kühn A, Valerius $\mathrm{O}$, Landesfeind $\mathrm{M}$, Aßhauer $\mathrm{K}$, Tech $\mathrm{M}$, et al. 2014. Verticillium transcription activator of adhesion Vta2 suppresses microsclerotia formation and is required for systemic infection of plant roots. New Phytologist. doi: 10.1111/nph.12671.

Travers KJ, Patil CK, Wodicka L, Lockhart DJ, Weissman JS, Walter P. 2000. Functional and genomic analyses reveal an essential coordination between the unfolded protein response and ER-associated degradation. Cell. doi: 10.1016/S00928674(00)80835-1.

Uttaro AD. 2006. Biosynthesis of polyunsaturated fatty acids in lower eukaryotes. IUBMB Life. doi: 10.1080/15216540600920899.

Vallad GE, Subbarao K V. 2008. Colonization of resistant and susceptible lettuce cultivars by a green fluorescent protein-tagged isolate of Verticillium dahliae. Phytopathology. doi: 10.1094/PHYTO-98-8-0871.

Wang RJ, Peng J, Li QX, Peng YL. 2017. Phosphorylation-mediated regulatory networks in mycelia of Pyricularia oryzae revealed by phosphoproteomic analyses. Molecular and Cellular Proteomics. doi: 10.1074/mcp.M116.066670.

Wilhelm S. 1955. Longevity of the Verticillium wilt fungus in the laboratory and field. Phytopath 45: 180-181.

Wilson RA, Calvo AM, Chang P-K, Keller NP. 2004. Characterization of the 
900

901

902

Aspergillus parasiticus 12-desaturase gene: a role for lipid metabolism in the Aspergillus-seed interaction. Microbiology. doi: 10.1099/mic.0.27207-0.

Yi M, Chi M-H, Khang CH, Park S-Y, Kang S, Valent B, Lee Y-H. 2009. The ER chaperone LHS1 is involved in asexual development and rice infection by the blast fungus Magnaporthe oryzae. The Plant Cell online. doi: 10.1105/tpc.107.055988.

Yu J, Li T, Tang C, Klosterman SJ, Tian C, Wang Y. 2019. Two Verticillium dahliae MAPKKKs, VdSsk2 and VdSte11, have distinct roles in pathogenicity, microsclerotial formation, and stress adaptation. mSphere. doi: 10.1080/07060661.2011.579173.

Zuker M. 2003. Mfold web server for nucleic acid folding and hybridization prediction. Nucleic acids research. doi: 10.1093/nar/gkg595.

\section{Figure legends}

Figure 1: The $V$. dahliae HAC1 gene of the unfolded protein response (UPR). (a) Verticillium dahliae HAC1 mRNA and protein variants. Unspliced HAC1 pre-mRNA (1634 nt) contains a conventional (53 nt, black) and an unconventional (20 nt, blue) intron. HAC1 produces two mRNA splice variants: Splicing of only the conventional intron results in HAC1u mRNA (1581 nt) for the potential Hac1u protein (526 aa); additional unconventional splicing results in the shorter induced HAC1' ORF (1254 nt), encoding the 417 aa Hac1 protein. Both proteins possess identical N-termini (268 aa) with basic leucine zipper domain (bZIP, grey, PS50217; 107-164 aa), and nuclear localization signal (NLS, red, 94 to $105 \mathrm{aa}$ ), whereas Hac1 ${ }^{\mathrm{u}}$ and Hac1 C-termini are unique (dark vs. light green). (b) Alignment of $5^{\prime}$ and $3^{\prime}$ splice sequences of unconventionally spliced introns shows high conservation of $V$. dahliae HAC1, Trichoderma reesei hac1 (M419DRAFT_128619), Aspergillus fumigatus hacA (XM_743634), Aspergillus nidulans hacA (AN9397), Alternaria brassicicola HacA (Joubert et al., 2011), Sacharomyces cerevisiae hac1 (NC_001138.5), Ustilago maydis cib1 (UMAG_11782) and Homo sapiens XBP1 (NM_005080.3)

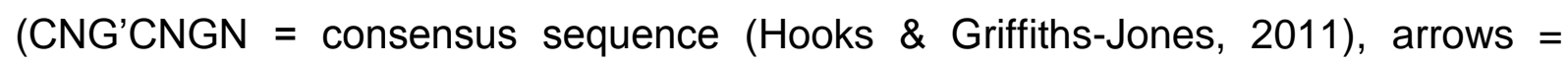
cleavage sites, lowercase characters $=$ intron sequences, capital letters = splice sequences, numbers of nucleotides are given for those not shown). (c) Predicted twin stem-loop secondary structures of $5^{\prime}$ and $3^{\prime}$ splice sequences of unconventional V. dahliae and S. cerevisiae HAC1 introns. (Arrows = cleavage sites, bold characters = splice sequences, lowercase characters = introns, $/ /=$ discontinuation of intron sequence) (d) Phylogenetic tree derived of Hac1-like proteins with Verticillium dahliae Hac1 (Table S5), Trichoderma reesei HACl (XP_006964054.1), Aspergillus fumigatus 


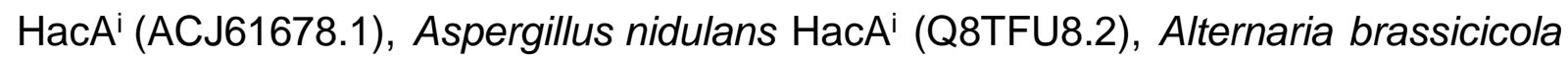
AbHacA (Joubert et al., 2011), Saccharomyces cerevisiae HAC1'i (NP_116622.1), Ustilago maydis cib1s $\quad$ (XP_011390112.1), and Homosapiens XBP1 (NP_001073007.1) sequences (Muscle algorithm, scale bar = average number of amino acid substitutions per site).

Figure 2: V. dahliae HAC1 supports growth and is essential for microsclerotia formation. Verticillium dahliae wildtype (WT), HAC1 deletion ( $\triangle H A C 1)$, and complementation $(H A C 1-C)$ strains, as well as strains expressing ectopically integrated HAC1 mRNA splice variants fused to $H A$ at the $3^{\prime}$-end in the $\triangle H A C 1$ strain (HAC14-HA; HAC1 $\left.{ }^{-}-H A\right)$ were compared. (a) Quantification of HAC1 gene expression by primers targeting both HAC1 mRNA splice variants. Mean values of two independent experiments with standard deviations normalized to wildtype and reference genes $H 2 A$ and $E I F 2 B$ are shown $\left({ }^{* *} \mathrm{p}<0.01\right.$, ns $=$ non-significant). $H A C 1$ gene expression is decreased in $H A C 1^{u_{-}} H A$ and $H A C 1^{i}-H A$. (b) Detection of Hac1 proteins in immunoblots using $\mathrm{HA}$-specific antibodies. Ponceau $\mathrm{S}$ staining served as a loading control. A strong signal is only visible at $\sim 70 \mathrm{kDa}$ for tagged Hac1 in the constitutively spliced $H A C 1^{i}-H A$ strain instead of the predicted $46 \mathrm{kDA}$, whereas there was no specific band for the predicted Hac1 $1^{\mathrm{u}}$-HA protein (59 kDa). (c) Microsclerotia formation ex planta. Wildtype-like microsclerotia were formed by all strains except $\triangle H A C 110 \mathrm{~d}$ after spot inoculation on CDM with sucrose with or without tunicamycin $(\mathrm{TM}=1 \mu \mathrm{g} / \mathrm{ml})$. Neither melanized nor unmelanized microsclerotia were observed for $\triangle H A C 1$ in cross sections of the colony centres (red boxes/dashed lines) or microscopy of fungal material (bottom). Ectopically expressed HAC1-C and HAC1 ${ }^{-} H A$ strains produce increased levels (Black scale bar $=1 \mathrm{~mm}$, yellow scale bar $=20 \mu \mathrm{m}$ ). $(\mathbf{d}$ ) Quantification of vegetative growth $10 \mathrm{~d}$ after spot inoculation. $\triangle H A C 1$ displayed reduced growth that is restored to wildtype-levels in $H A C 1-C$ and $H A C 1_{-}-H A$, whereas HAC1-HA displayed a decrease in growth under non-stress conditions and increased growth upon supplementation of tunicamycin (TM). Mean values of three independent experiments with standard deviations relative to wildtype are shown $\quad\left({ }^{*} p<0.05\right.$; ${ }^{* *} \mathrm{p}<0.01 ;{ }^{* *} \mathrm{p}<0.001,{ }^{* * *} \mathrm{p}=0$, ns $=$ non-significant, $\left.\mathrm{n} \geq 2\right)$.

Figure 3: V. dahliae HAC1 is required for initial plant root colonization, conidia 956 formation, and induction of disease symptoms in tomato plants. Verticillium dahliae wildtype (WT), HAC1 deletion ( $\triangle H A C 1)$, and complementation (HAC1-C) 
strains, as well as wildtype and $H A C 1$ deletion strains constitutively expressing ectopic GFP (WT OE-GFPNAT, $\triangle H A C 1$ OE-GFP) were compared. (a) V. dahliae colonization of Arabidopsis thaliana roots. Fluorescence confocal microscopy was performed $7 \mathrm{~d}$ post inoculation of roots with the same numbers of spores from wildtype WT OEGFPNAT or $\triangle H A C 1 O E-G F P$ with four plants per strain in two independent experiments. $\triangle H A C 1$ OE-GFP displays reduced propagation on the root surface, but was able to penetrate roots (white arrows) and colonize the root cortex (Scale bar $=20 \mu \mathrm{m}$ ). (b) Quantification of conidiation $5 \mathrm{~d}$ post inoculation of liquid simulated xylem medium revealed reduced $\triangle H A C 1$ conidiation compared to wildtype. Mean values of four independent experiments with standard deviations relative to wildtype are shown $\left({ }^{* * *} \mathrm{p}\right.$ $=0$, ns = non-significant, $\mathrm{n} \geq 4$ ). (c) Pathogenicity test of $\triangle H A C 1$ mutant compared to wildtype towards Solanum lycopersicum. Representative plants and hypocotyl dissections $21 \mathrm{~d}$ after root dipping into distilled water control (Mock), or same numbers of spores obtained from different strains are shown (Scale bar $=1 \mathrm{~mm}$ ). Relative amount of plants with certain disease scores from two independent experiments are displayed in the stack diagram ( $\mathrm{n}=$ total number of evaluated plants). $\triangle H A C 1$ treated plants showed mock-like hypocotyl coloration, disease symptoms in only few plants and no fungal outgrowth from surface sterilized stem sections after $7 \mathrm{~d}$ (bottom).

Figure 4: Pheromone response mitogen activated protein (MAP) kinase module of $\boldsymbol{V}$. dahliae. Intron-exon boundaries and resulting open reading frames (ORF) were confirmed by PCR amplification and sequencing of wildtype cDNA. (a) Architecture of the V. dahliae MAPK module including Ham5 scaffold. (b) Transcript structures and deduced protein kinase domains of $V$. dahliae MAPK2 Mek2 (left) and MAPK Vmk1 (right). The Mek2 ORF results in 522 amino acids (aa) with protein kinase domain (blue bar: 67-332 aa; IPR000719) including active serine/threonine site residues (grey: 186198aa, IPR008271) and ATP binding site (red: 73-96 aa; IPR017441). Vmk1 is smaller and consists of 355 aa (blue: protein kinase domain: 23-311 aa, IPR000719; grey: active site serine/threonine residues: 143-155 aa, IPR008271; red: ATP binding site: 29-53aa; IPR017441). (c) HAM5 (VDAG_JR2_Chr4g07170a) transcripts and deduced protein domains. The 1553 aa Ham5 scaffold contains WD40 repeats at the N-terminus (black: 10-265 aa, IPR015943) and a coiled-coil domain (blue: 1154-1182 aa). Related Ham5-like proteins of other fungi are depicted in a phylogenetic tree (ClustalW algorithm) with Trichoderma reesei HAM-5 (AKN58846.1), Neurospora crassa HAM-5 (XP_011393509.1), Podospora anserina IDC1 (ABJ96338.2), Aspergillus nidulans 
HamE (AN2701), and Saccharomyces cerevisiae Ste5 (NP_010388.1) $($ Scale bar = average number of amino acid substitutions per site).

Figure 5: V. dahliae Vmk1 and Mek2 kinases but not the Ham5 scaffold are required for efficient melanization and virulence. The HAM5 deletion strain ( $\triangle H A M 5)$ was compared to wildtype (WT), VMK1 and MEK2 single deletions ( $\triangle V M K 1$; $\triangle M E K 2)$ with respective complementation strains (VMK1-C; MEK2-C), as well as $\triangle H A M 5 \triangle V M K 1$ and $\triangle H A M 5 \triangle M E K 2$ double deletions. (a) Vegetative growth and melanization ex planta. 50000 freshly harvested spores were spot inoculated on CDM with either sucrose or cellulose and incubated at $25^{\circ} \mathrm{C}$ for $9 \mathrm{~d}$. Cross sections of colony centres (red boxes/dashed lines) and microscopy of fungal material scrapped from colony centres grown on CDM with cellulose are shown (black scale bar: $1 \mathrm{~mm}$, blue scale bar: $20 \mu \mathrm{m}$ ). (b) left: Growth quantification $9 \mathrm{~d}$ after spot inoculation on CDM with sucrose or cellulose. $\triangle H A M 5$ displays wildtype-like growth, whereas other single and double deletion strains are slightly repressed in growth. Mean values of three independent experiments with standard deviations relative to wildtype are shown $\left({ }^{*} p<0.05 ;{ }^{* *} p<0.01 ;{ }^{* * *} p<0.001,{ }^{* * * *} p=0, n \geq 3\right)$. right: Melanization of colonies grown on CDM with cellulose relative to wildtype. Whereas $\triangle H A M 5$ showed wildtype-like melanization, other single and double deletion strains displayed a decrease to about $40 \%$. Mean values of two independent experiments with standard deviations relative to wildtype are shown $\left({ }^{*} p<0.05 ;{ }^{* \star *} p<0.001,{ }^{* * * *} p=0\right.$, ns $=$ non-significant, $\left.n \geq 2\right)$. (c) Pathogenicity test of MAPK pathway mutants towards Solanum lycopersicum. Representative plants and hypocotyl dissections $21 \mathrm{~d}$ after root dipping into distilled water as control (Mock), or the same numbers of spores obtained from different strains are shown (Scale bar $=1 \mathrm{~mm}$ ). The relative number of plants with certain disease scores are displayed in the stack diagram (total number of evaluated plants $=n$ ). $\triangle H A M 5$ induced wildtype-like disease symptoms, whereas $\triangle M E K 2, \triangle V M K 1$, $\triangle H A M 5 \triangle M E K 2$, or $\triangle H A M 5 \triangle V M K 1$ treated plants were comparable to mock plants, and show no hypocotyl discolorations.

Figure 6: V. dahliae Ode1 is localized to plasma and nuclear membranes. The $O D E 1-G F P$ strain harbours a functional fusion at the endogenous locus under control of the native promoter and terminator. This strain with or without RFP tagged histone $\mathrm{H} 2 \mathrm{~B}$, and wildtype constitutively expressing free GFP (WT OE-GFPHYG) or RFP tagged histone H2B (JR2 Histone-RFP) were compared. (a) Verticillium dahliae oleate $\Delta 12$ - 
1025 fatty acid desaturase encoding ODE1 intron-exon boundaries (1504 bp) were

1026

1027

1028

1029

1030

1031

1032

1033

1034

1035

1036

1037

1038

1039

1040

1041

1042

1043

1044

1045

1046

1047

1048

1049

1050

1051

1052

1053

1054

1055

1056

1057 confirmed by PCR amplification from wildtype cDNA and sequencing. The deduced Ode1 protein (481 aa) contains two fatty acid desaturase domains (FAD; yellow; 77-112 aa, IPR021863; 138-424 aa; IPR005804), and four putative transmembrane helices (grey; 105-124 aa, 136-157 aa, 300-319 aa, 331-350 aa; Phobius). (b) Scheme of the predicted Ode1 protein structure. The $\mathrm{N}$ - and $\mathrm{C}$-termini of the transmembrane protein are directed to the cytosol. An iron atom (Fe) and the FAD domains (yellow) form the catalytic centre. (c) Detection of Ode1-GFP (81 kDa) in immunoblot analysis using GFP-specific antibody. Ponceau S staining served as loading control. (d) Fluorescence microscopy revealed Ode1-GFP subcellular localization to membranes of round cell organelles and close to hyphal tips (white arrow) of growing hyphae $12 \mathrm{~h}$ after inoculation (Scale bar = $10 \mu \mathrm{m}$ ). (e) Ode1-GFP is not localized to FM4-64 red stained vacuole membranes $16 \mathrm{~h}$ post inoculation (scale bar $=10 \mu \mathrm{m}$ ). (f) Ode1-GFP is localized to membranes surrounding red nuclei in the ODE1-GFP Histone-RFP strain. Fluorescence microscopy $16 \mathrm{~h}$ post inoculation (yellow scale bar $=20 \mu \mathrm{m}$, white scale bar $=10 \mu \mathrm{m})$.

Figure 7: V. dahliae ODE1 contributes primarily to vegetative growth with only minor effects on plant disease symptoms. The Verticillium dahliae ODE1 deletion strain ( $\triangle O D E 1)$ was compared to wildtype (WT) and the complementation strain harbouring ODE1-GFP at the endogenous locus under control of the native promoter and terminator. (a) Vegetative growth and microsclerotia formation ex planta $9 \mathrm{~d}$ after spot inoculation on CDM supplemented with $\mathrm{NaCl}(0.5 \mathrm{M}), \mathrm{CDM}$ with either sucrose or cellulose, and CDM with cellulose supplemented with linoleic acid (LA, $0.125 \mathrm{mg} / \mathrm{ml}$ ). $\triangle O D E 1$ formed smaller colonies on all tested media. Cross sections of colonies grown on CDM with cellulose (red boxes/ dashed lines) and microscopy of fungal material (bottom) showed wildtype-like microsclerotia formation of $\triangle O D E 1$ (black scale bar = $1 \mathrm{~mm}$, yellow scale bar $=20 \mu \mathrm{m}$ ). (b) Growth quantification 3, 6, and $9 \mathrm{~d}$ after spot inoculation on CDM with cellulose with or without linoleic acid (LA, $0.125 \mathrm{mg} / \mathrm{ml}$ ). $\triangle O D E 1$ displayed about $50 \%$ decreased growth $9 \mathrm{~d}$ after spot inoculation on CDM with cellulose. LA supplementation partially complements the growth defect. Mean values and standard deviations relative to wildtype of two independent experiments are shown $\left({ }^{* *} \mathrm{p}<0.01 ;{ }^{* *} \mathrm{p}<0.001,{ }^{* * *} \mathrm{p}=0, \mathrm{n} \geq 3\right)(\mathbf{c})$ Pathogenicity test of ODE1 mutants towards Solanum lycopersicum. Representative plants and hypocotyl dissections $21 \mathrm{~d}$ after 
1058

1059

1060

1061

1062

1063

1064

1065

1066

1067

1068

1069

1070

1071

1072

1073

1074

1075

1076

1077

1078

1079

1080

1081

1082

1083

1084

1085

1086

1087

root dipping into distilled water control (Mock), or same number of spores obtained from different strains are shown (Scale bar $=1 \mathrm{~mm}$ ). Relative amount of plants with certain disease scores from three independent experiments are displayed in the stack diagram ( $\mathrm{n}=$ total number of evaluated plants). $\triangle O D E 1$ infection resulted in only a minor decrease in disease symptom induction.

Figure 8: Model of $V$. dahliae signalling pathways and their functions in development and induction of disease symptoms. (1) The unspliced mRNA of the UPR regulator Hac1 $\left(H A C 1^{4}\right.$ ) is unconventionally spliced (arrow close to ER membrane), and the $H A C 1^{i} \mathrm{mRNA}$ is translated into the bZIP transcription factor Hac1, which regulates UPR target genes. V. dahliae HAC1 is involved in the ER stress response and vegetative growth under non-stress conditions, has a strong impact on conidiation, and is essential for the formation of microsclerotia (arrow/ green plus to "Growth and development"). HAC1 is required for the virulence of $V$. dahliae (arrow/ red minus from fungal cell) and potentially regulates expression or secretion of effector proteins, which enables the fungus to circumvent plant defence responses (arrow with dashed line/ green plus from plant cell). (2) The $V$. dahliae Vmk1 MAPK pathway components Mek2 (MAP2K) and Vmk1 (MAPK) positively control vegetative growth and microsclerotia formation (arrow/ green plus to "Growth and development") and are required for induction of disease symptoms in plants (arrow/ red minus from fungal cell) independent from the scaffold homolog Ham5 in V. dahliae. (3) The oleate $\Delta 12$-fatty acid desaturase Ode1, which catalyses the synthesis of linoleic acid, supports fungal growth (arrow/ green plus to "Growth and development"). Ode1 deficiency has only minor effect on development of disease symptoms in planta, which might be due to the availability of plant linoleic acid to potentially to support fungal growth and differentiation (arrow with dashed line/ green plus from plant cell).

\section{Supporting Information}

Additional information may be found in the online version of this article.

\section{Supporting Information Figures}

Figure S1 Southern hybridization of $V$. dahliae HAM5, VMK1 and MEK2 single and double deletion, and complementation strains. 
1088 Figure S2 Southern hybridization of $V$. dahliae HAC1 deletion, HAC1 deletion with 1089 ectopic GFP overexpression, HAC1-C complementation, as well as $H A C 1^{u_{-}} H A$ and 1090 HAC14-HA strains.

1091 Figure S3 Southern hybridization of $V$. dahliae ODE1 deletion and ODE1-GFP 1092 complementation strains.

1093 Figure S4 Phylogeny of Rok1-like phosphatases.

1094 Supporting Information Tables

1095 Table S1 Verticillium strains constructed and used in this study.

1096 Table S2 Primers used in this study.

1097 Table S3 Plasmids constructed and used in this study.

1098 Table S4 The cDNA sequence of V. dahliae JR2 HAC1i.

1099 Table S5 The amino acid sequence of $V$. dahliae JR2 Hac1.

1100 Supporting Information Methods 


\section{V. dahliae}

HAC1

pre-mRNA

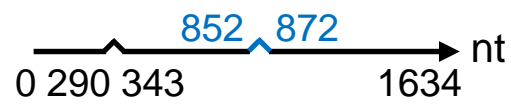

HAC1u
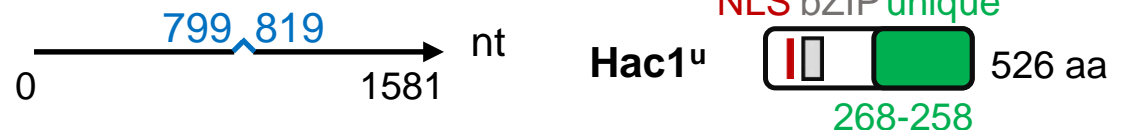

HAC1i

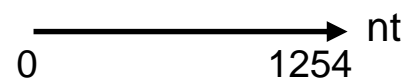

Hac1

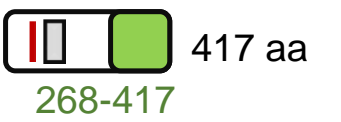

b

Consensus splice sites

V. dahliae

5' CNGengn

T. reesei<smiles></smiles>

A. fumigatus GUCCUGcagaga

8 nt- gaccugCCGUGU

CngCNGN 3"

A. nidulans

AUCCUGcagc 99 .

8 nt- gacccgCAGUGU

A. brassicicola

AUCCUGcagc 9

8 nt- gac cugCAGU

S. cerevisiae

AUCCUGccge
$A U C$ CAGccgu

8 nt- gaccugCAGU

U. maydis

8 nt- gaccugCCGU

H. sapiens

UCCCUGcagc

-240 nt-

CCgAAGC

C

S. cerevisiae hac1

V. dahliae HAC1
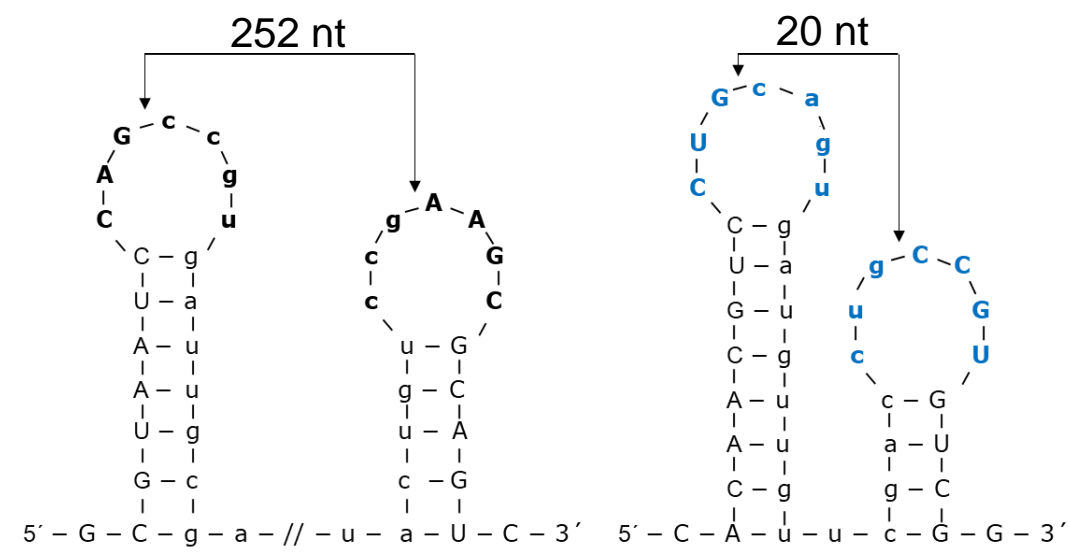

d

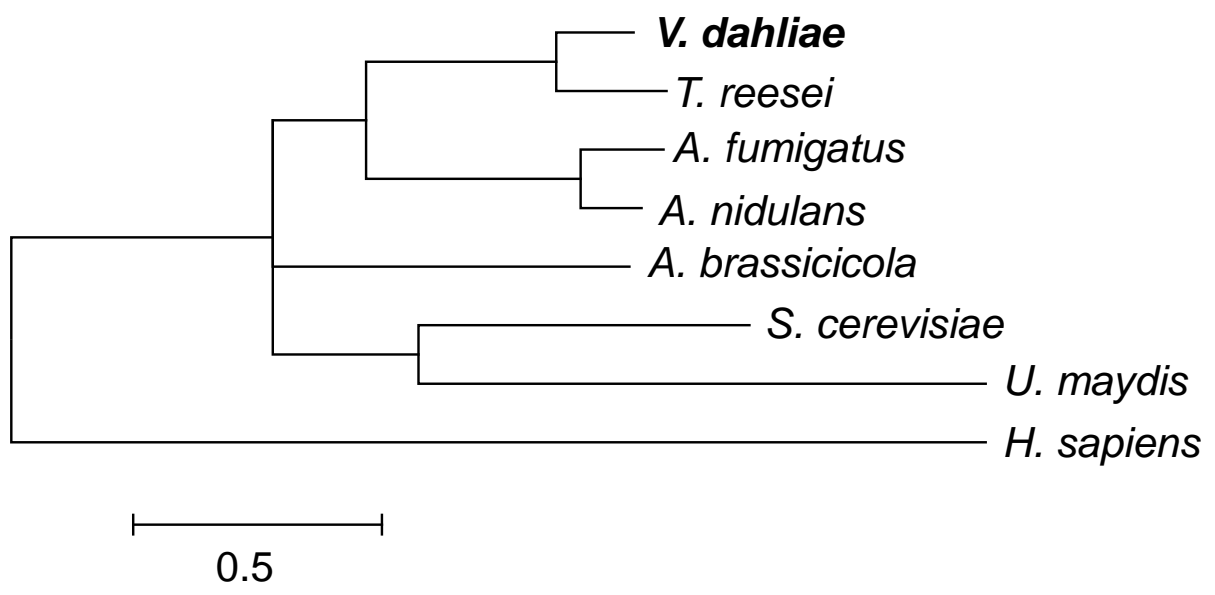

Figure 1 


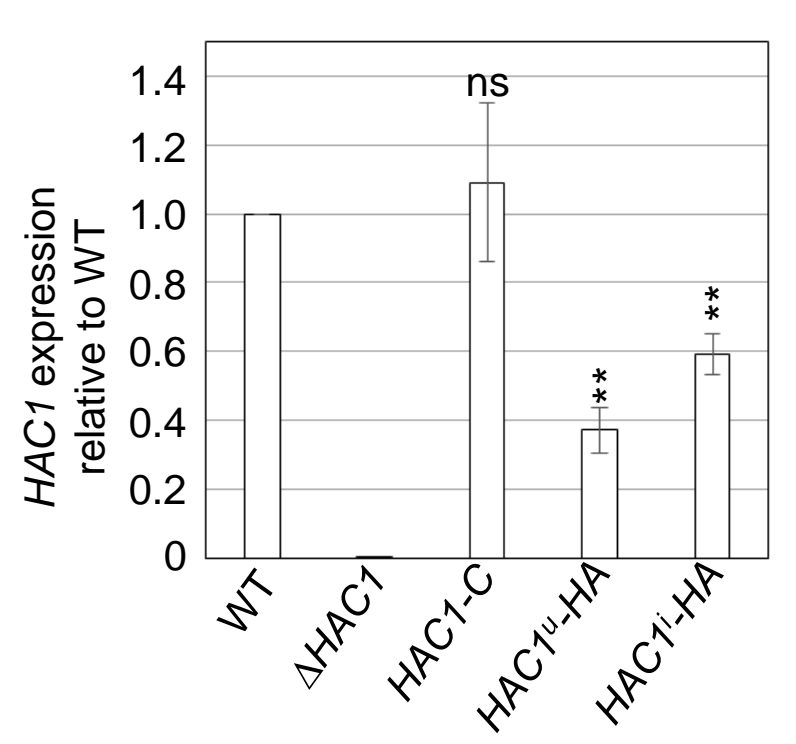

b

c WT $\triangle H A C 1$ HAC1-C HAC1U-HAHAC1i-HA

a

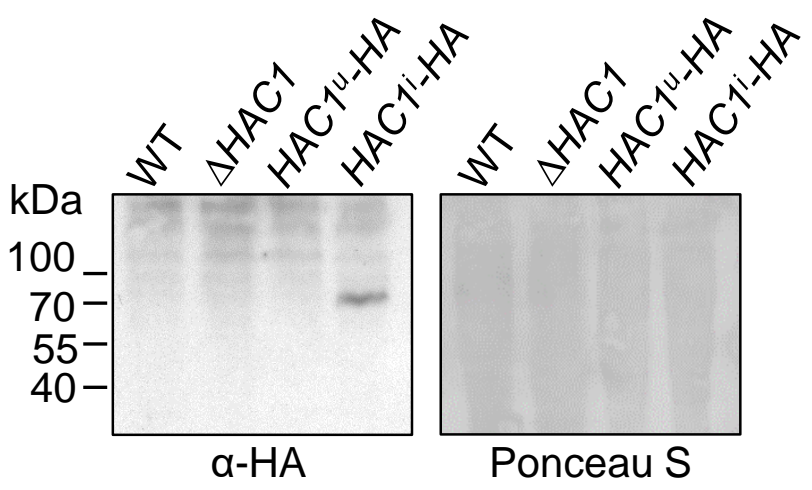

d $\quad \square C D M+$ sucrose D CDM+sucrose+TM
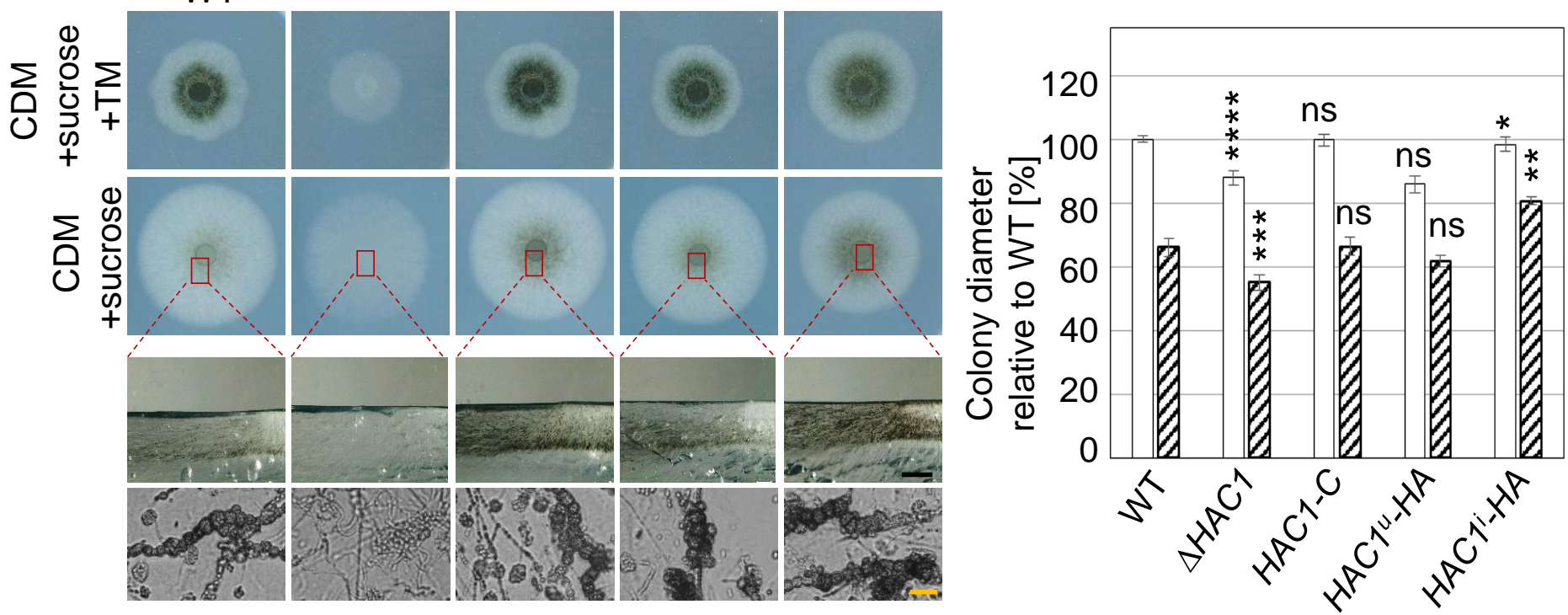

Figure 2 
a
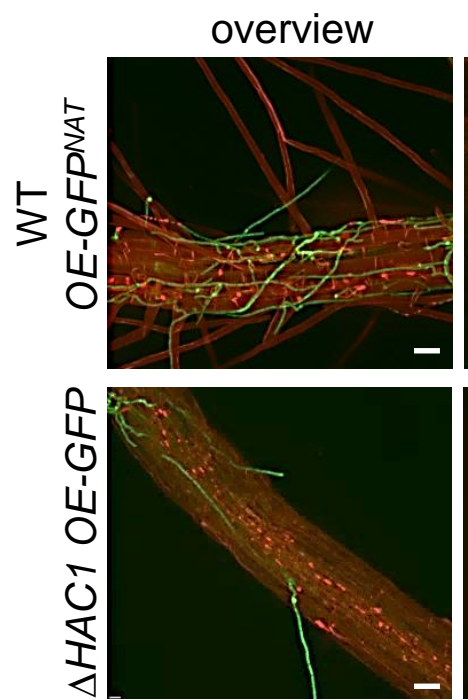

C

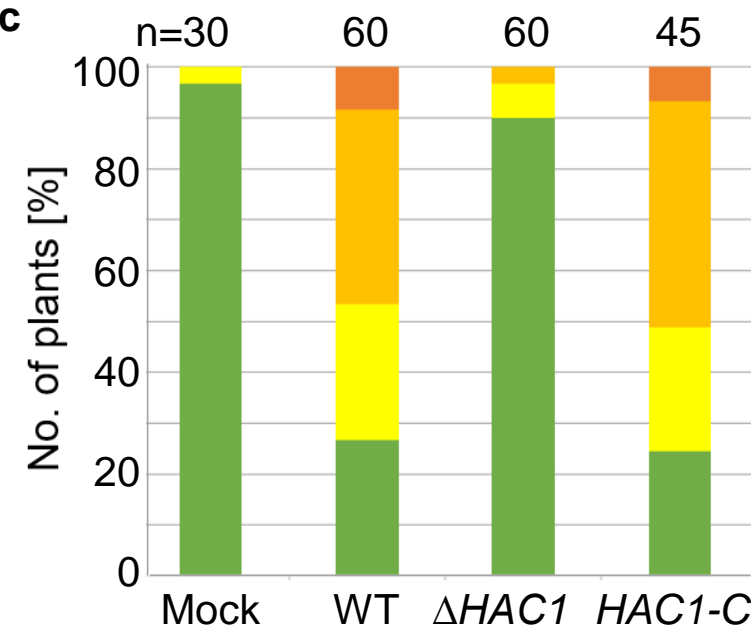

zoom in
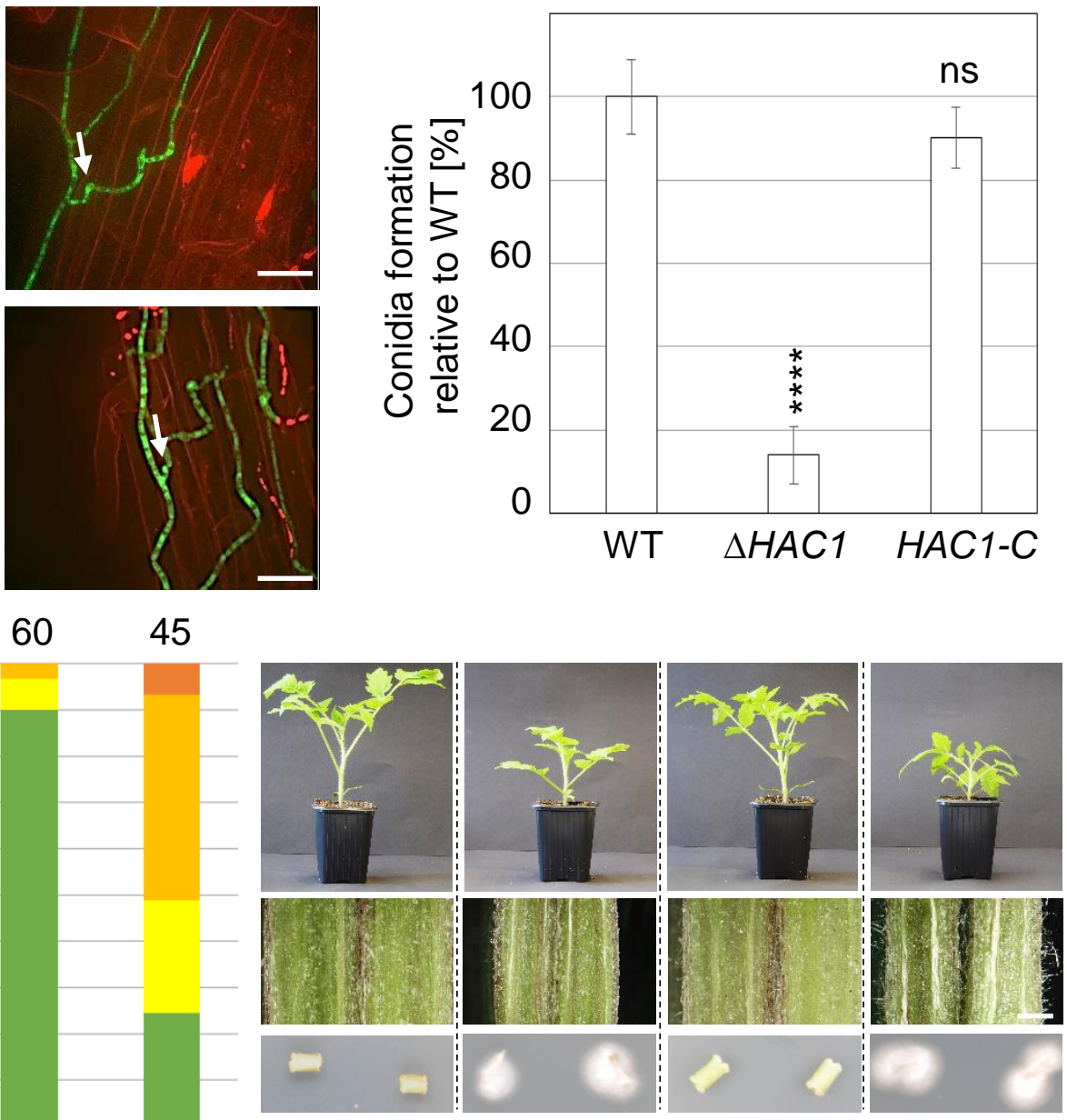

Mock

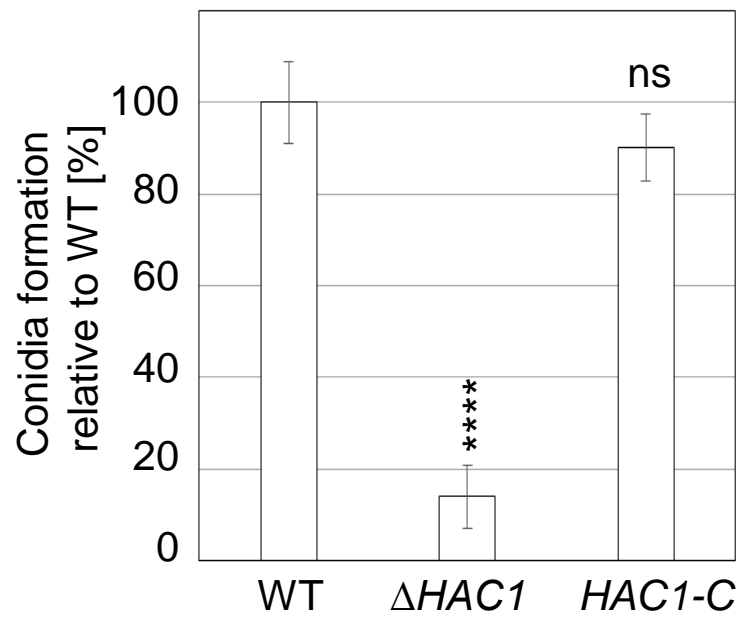

Disease scores:

Heavy symptoms Strong symptoms Weak symptoms Healthy plant

Figure 3 


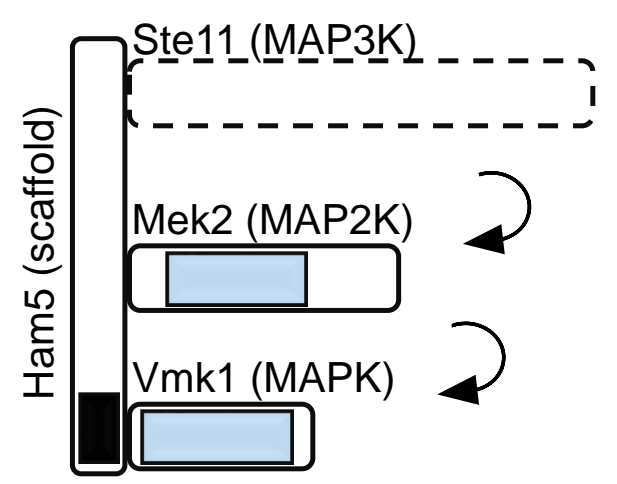

V. dahliae Mek2

pre-

mRNA

protein

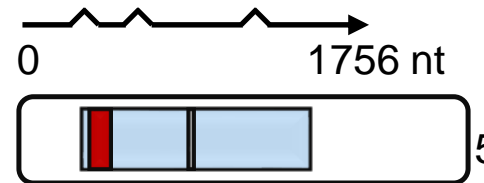
522 aa

Serine/threonine protein kinase

V. dahliae Vmk1 pre-

mRNA

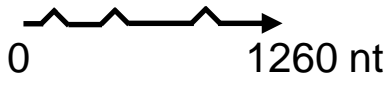
active site

protein

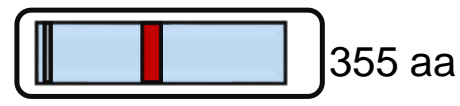

$\square$ Protein kinase domain

ATP binding site

C

V. dahliae Ham5 pre-mRNA
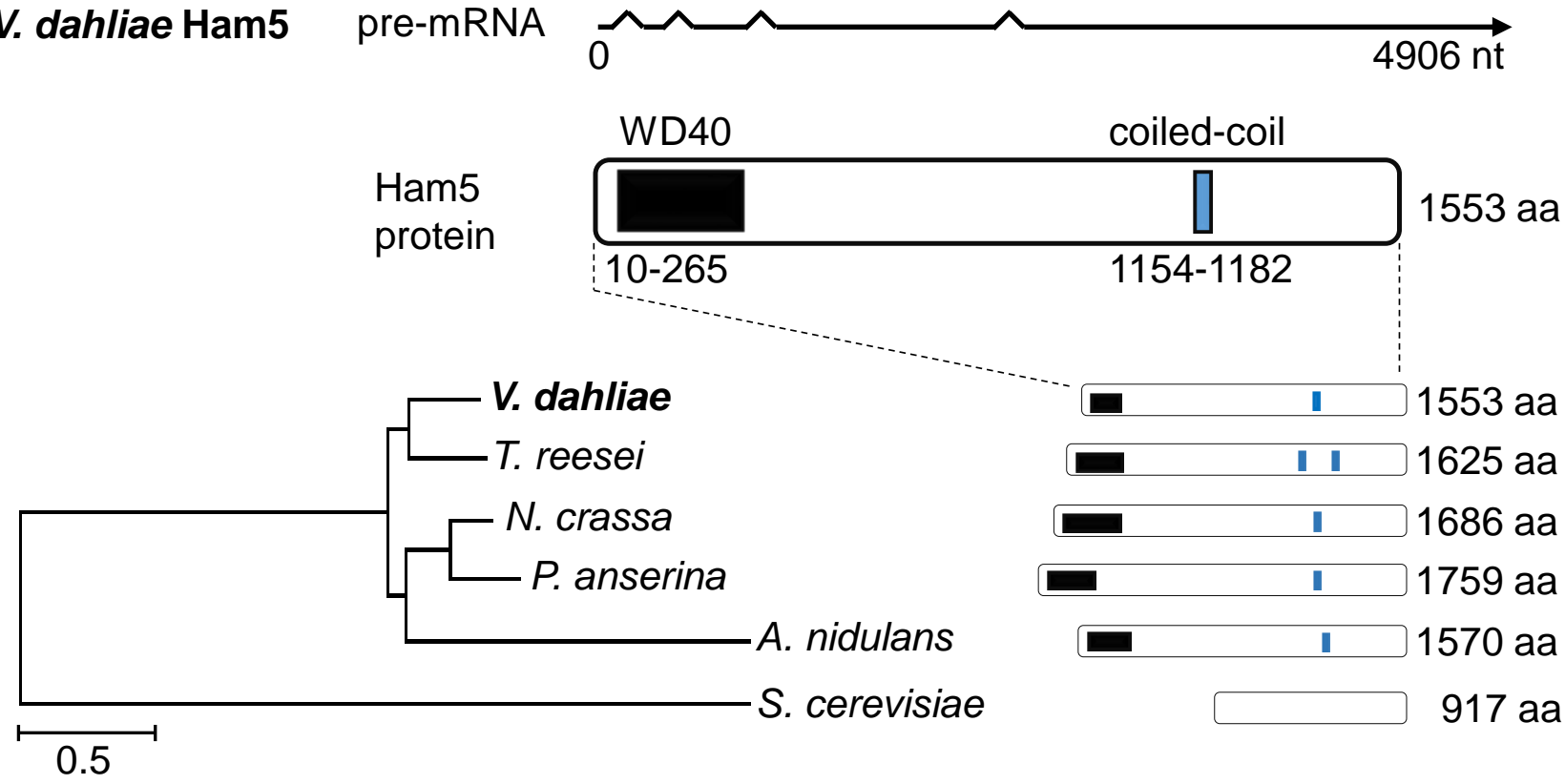

Figure 4 
$a$

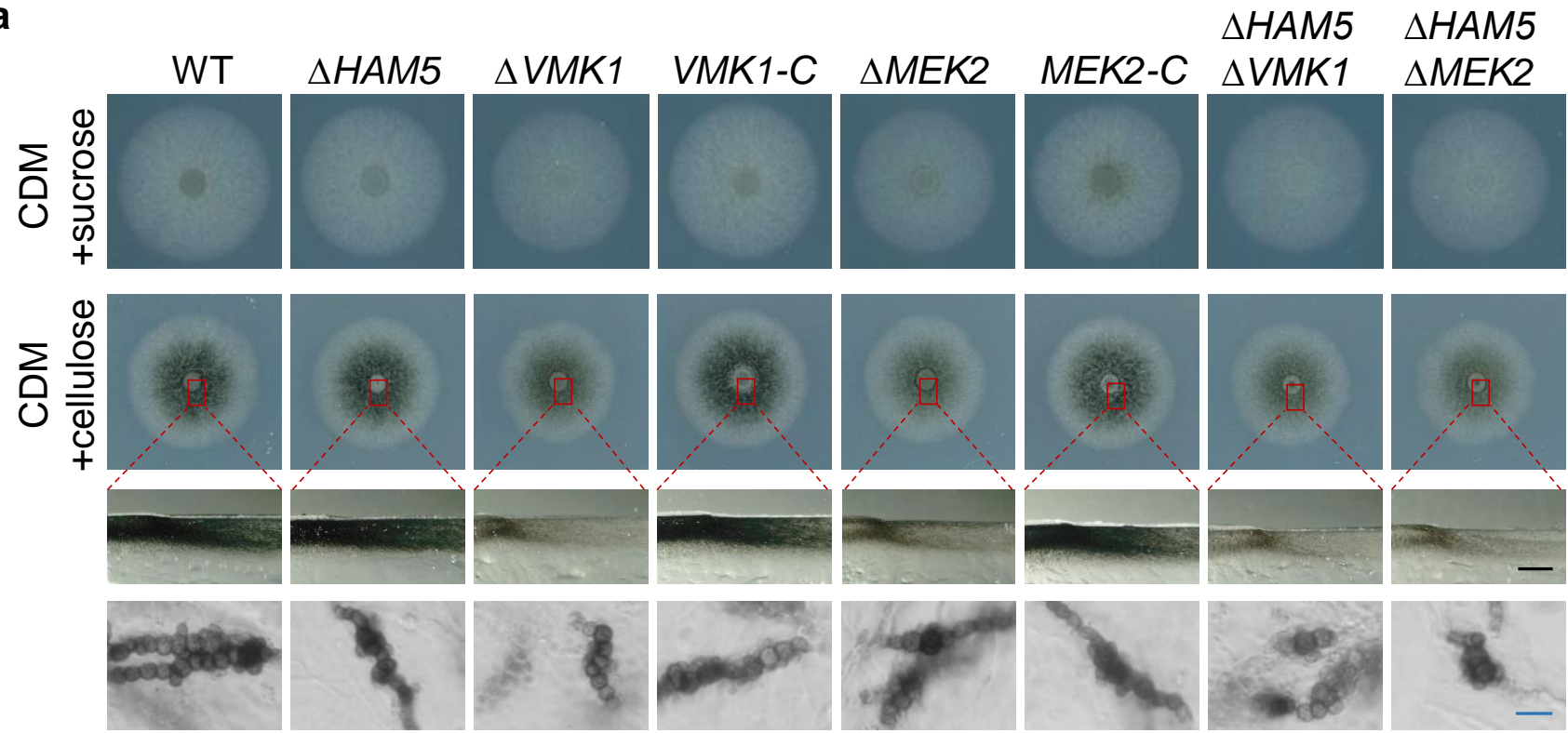

b

$\square$ CDM+sucrose CDM+cellulose
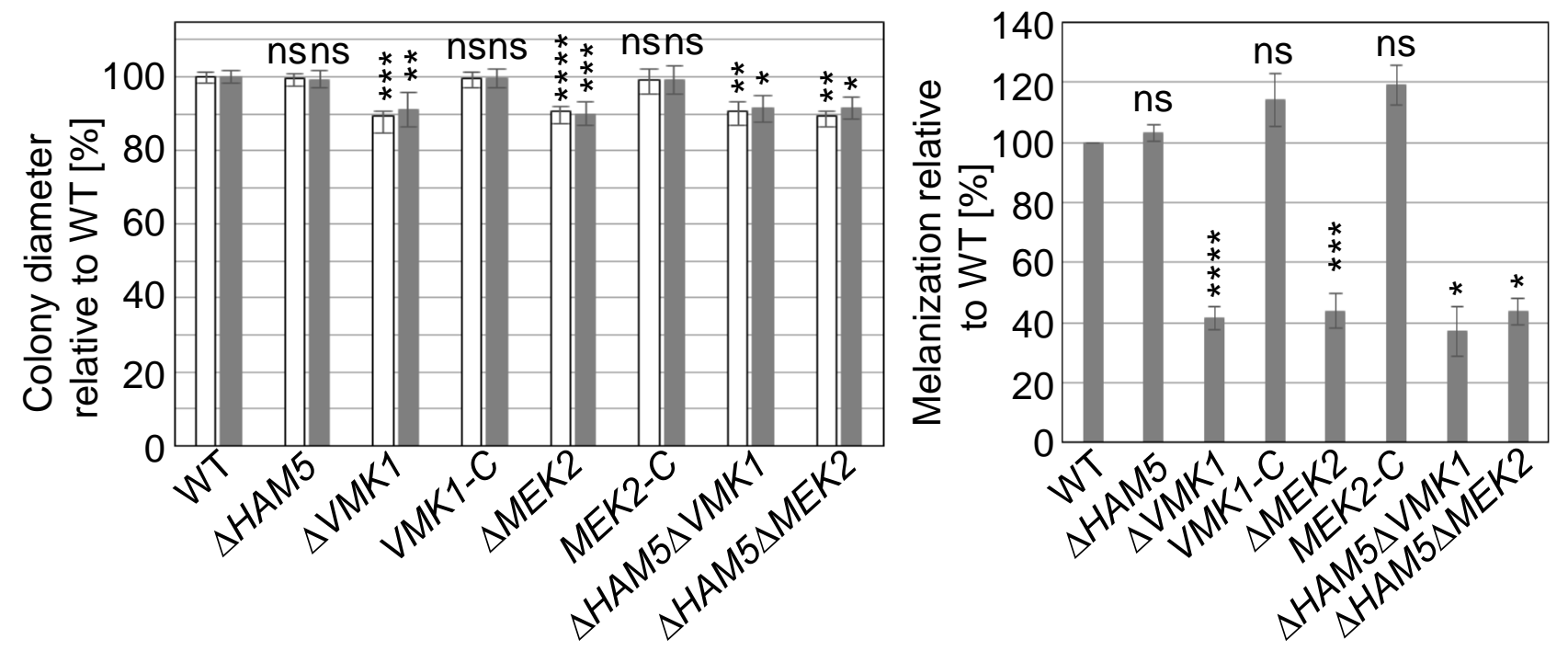

C
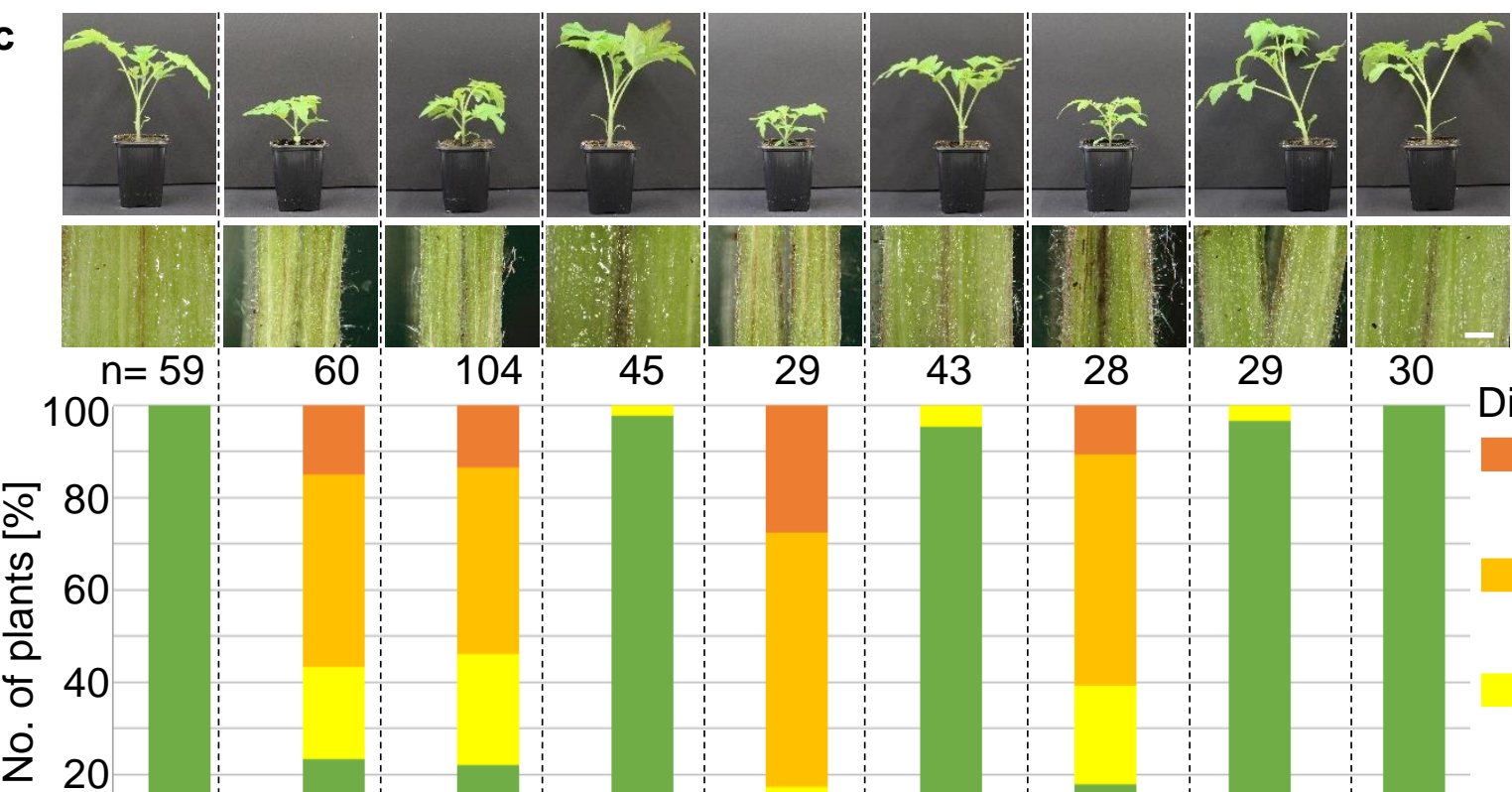

$45 \quad 29$
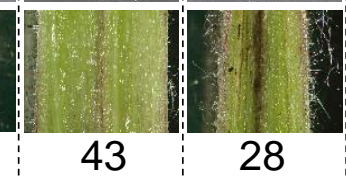

28

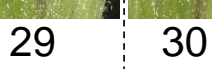

Disease scores:

Heavy Symptoms

Strong symptoms

Weak symptoms<smiles>C=CC(C)C</smiles>

Figure 5

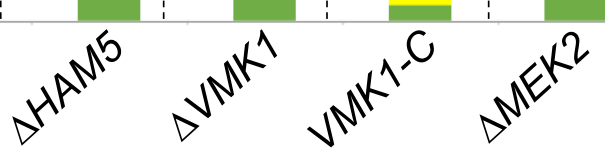

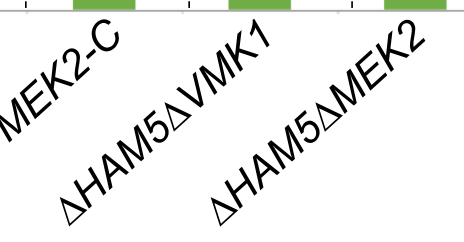

Healthy plant 
a

V. dahliae ODE1

pre-

mRNA 0

Ode1

protein

$\square$ Fatty acid desaturase domain

$\square$ Transmembrane helix

C

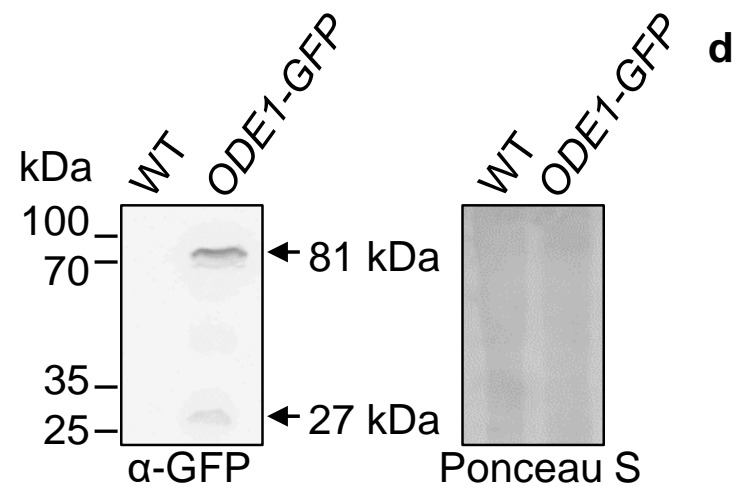

e

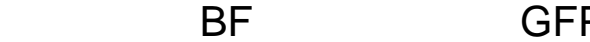

$\frac{\frac{0}{4}}{\frac{1}{4}} \frac{0}{0}$

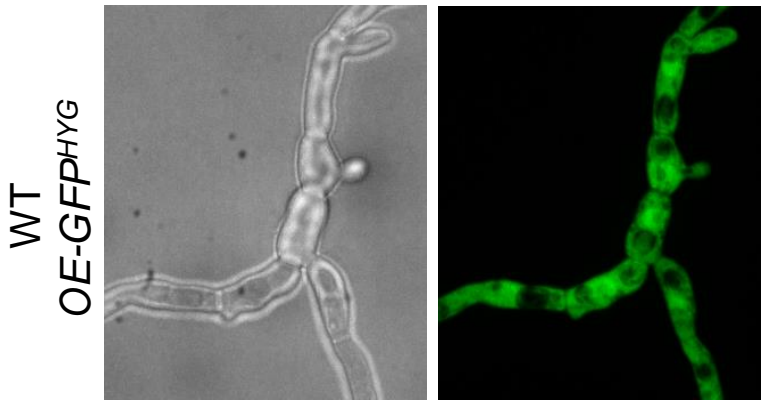

f

BF

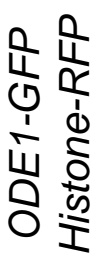

$\frac{0}{x}$

$\sum \stackrel{1}{\delta}$

Figure 6

b

Cytosol $481 \mathrm{aa}$

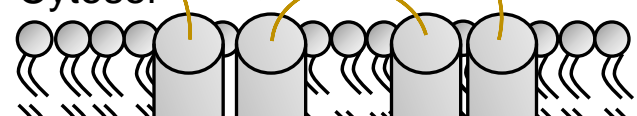

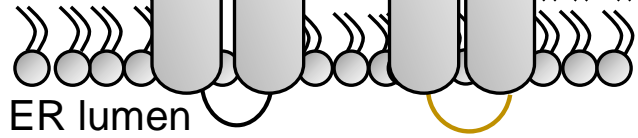

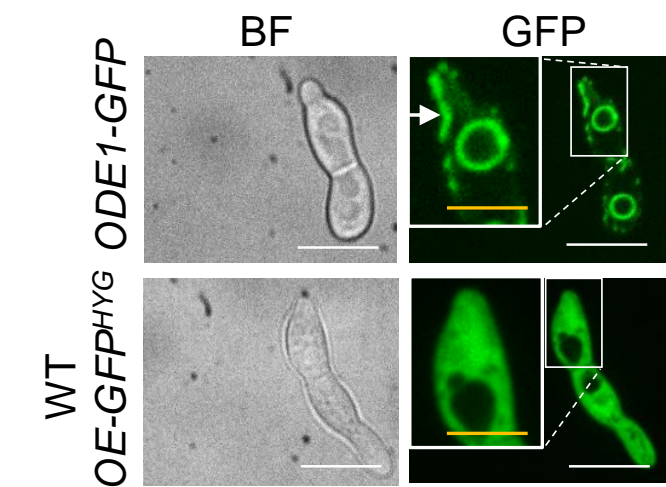

FM4-64

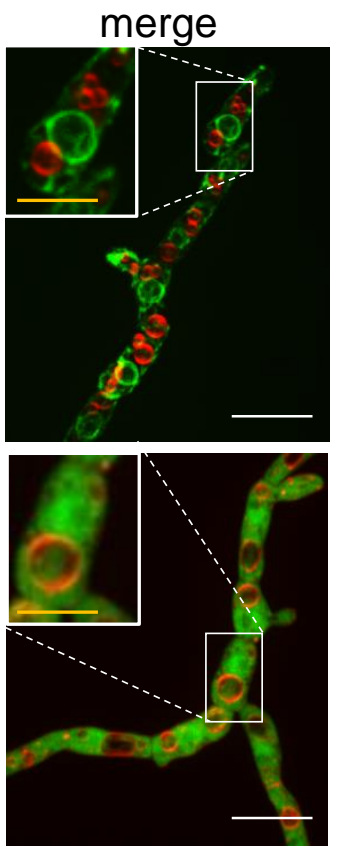

RFP

merge
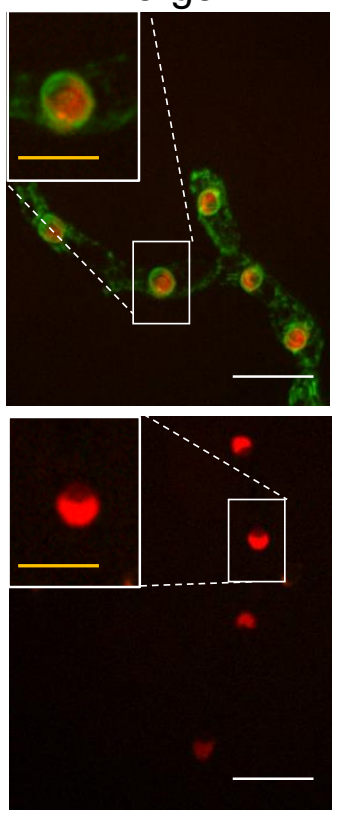
a

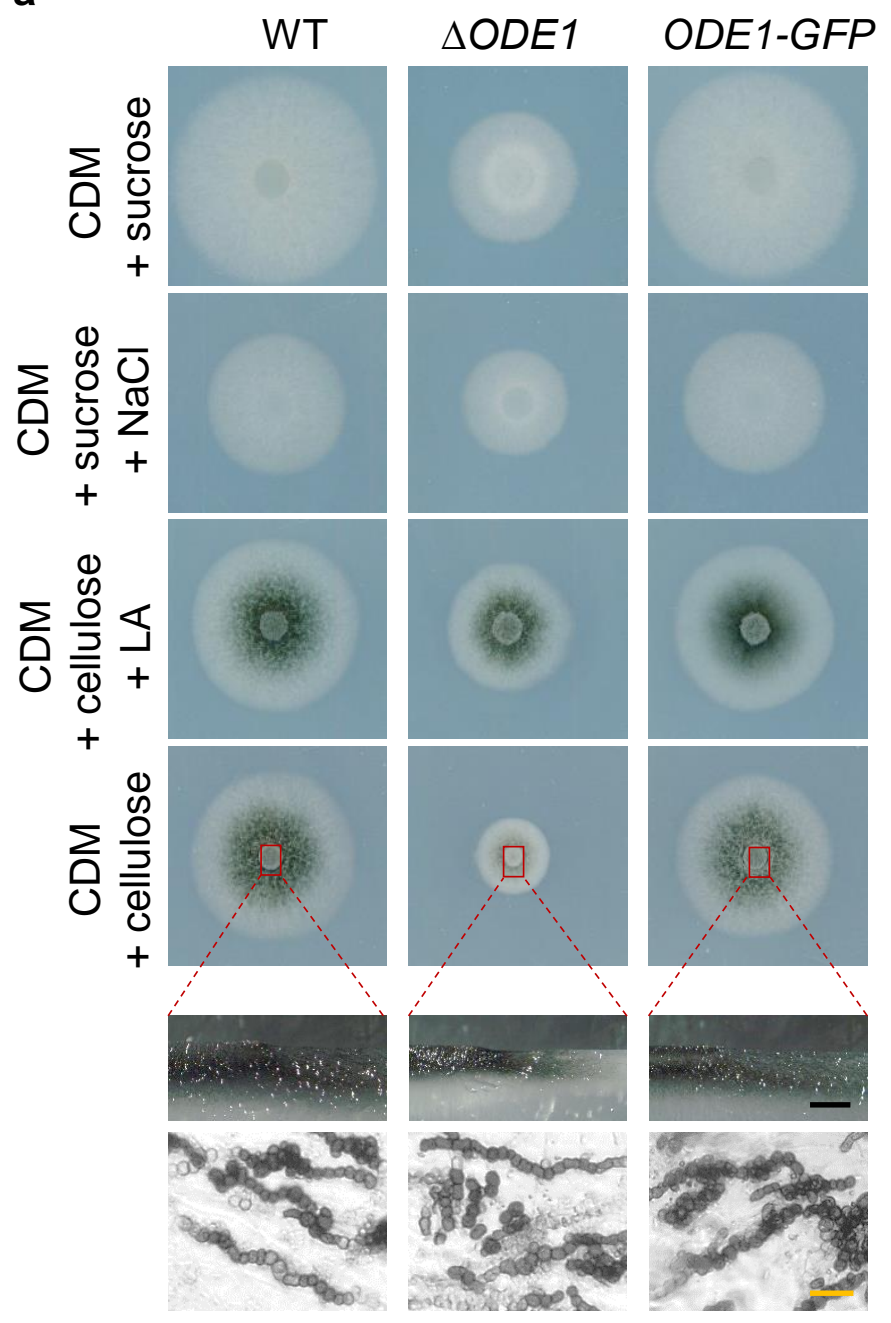

C

\section{Figure 7}
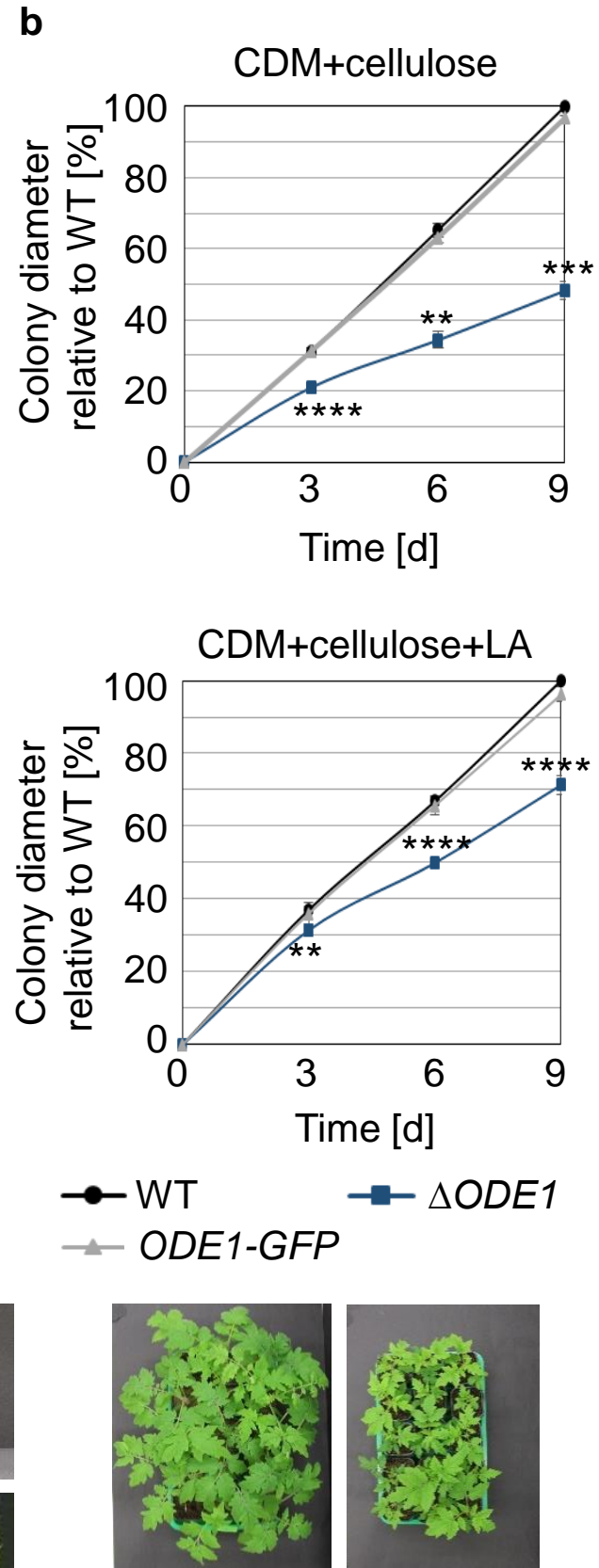

Mock

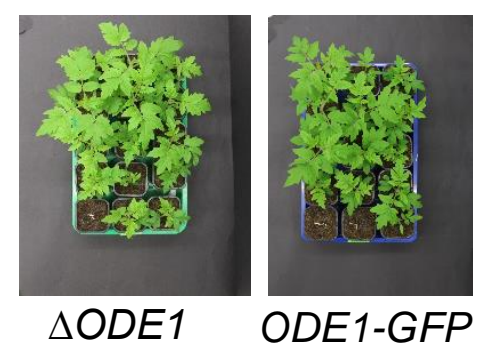

Disease scores:

Heavy symptoms

Strong symptoms Weak symptoms

Healthy plant 


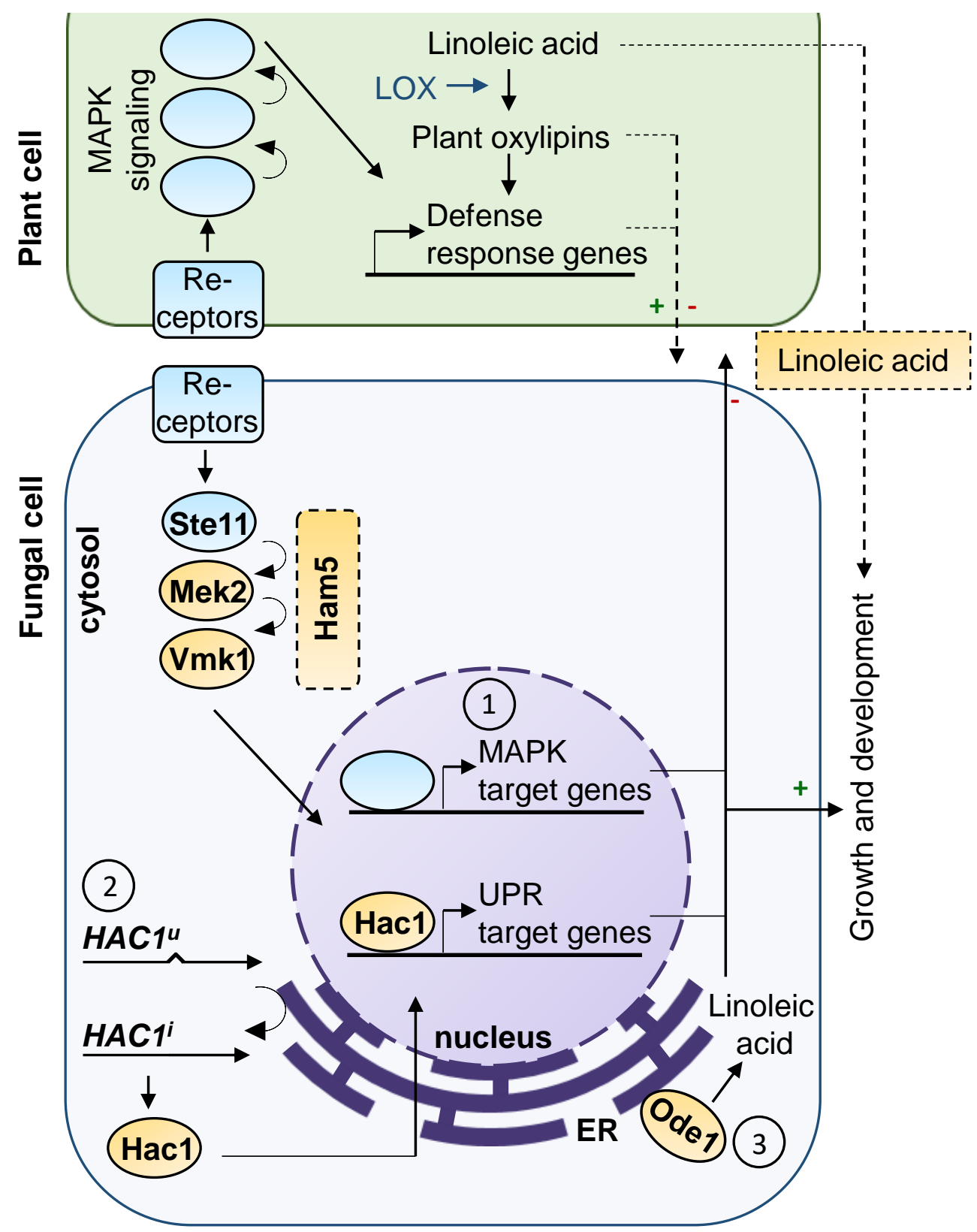

Figure 8 